\title{
A multilevel computational strategy for handling microscopic and macroscopic instabilities
}

\author{
S. Nezamabadi ${ }^{a}, J$. Yvonnet ${ }^{\mathrm{b}, *}$, H. Zahrouni ${ }^{\mathrm{a}}$, \\ M. Potier-Ferry ${ }^{\text {a }}$ \\ ${ }^{a}$ Université Paul Verlaine de Metz, Laboratoire de Physique et Mécanique des \\ Matériaux, UMR CNRS 7554, Ile du Saulcy 57045, Metz Cedex 01 France \\ ${ }^{\mathrm{b}}$ Université Paris-Est, Laboratoire Modélisation et Simulation Multi Échelle, FRE \\ CNRS 3160, 5 Bd Descartes, 77454 Marne-la-Vallée Cedex 2, France
}

\begin{abstract}
This paper presents a numerical technique to deal with instability phenomena in the context of heterogeneous materials where buckling may occur at both macroscopic and/or microscopic scales. We limit ourselves to elastic materials but geometrical nonlinearity is taken into account at both scales. The proposed approach combines the multilevel finite element analysis $\left(\mathrm{FE}^{2}\right)$ and the asymptotic numerical method (ANM). In that framework, the unknown nonlinear constitutive relationship at the macroscale is found by solving a local finite element problem at the microscale. In contrast with $\mathrm{FE}^{2}$, the use of the asymptotic development allows to transform the nonlinear microscopic problems into a sequence of linear problems. Thus, a direct analogy with classical linear homogenization can be made to construct a localisation tensor at each step of the asymptotic development, and an explicit macroscopic constitutive relationship can be constructed at each step. Furthermore, the salient features of the ANM allow treating instabilities and limit points in a very simple way at both scales. The method is tested and illustrated through numerical examples involving local instabilities which have significant influence on the macroscopic behavior.
\end{abstract}

Key words: Asymptotic numerical method, Nonlinear homogenization, Multilevel finite element method, Instabilities, Buckling

* Correspondence to J. Yvonnet

Email address: julien.yvonnet@univ-paris-est.fr (J. Yvonnet). 


\section{Introduction}

The understanding and modelling of instability phenomena in heterogeneous materials containing dispersions of multiple phases in the microstructure is of critical importance to design advanced materials in high performance applications. To give the macroscopic overall response of heterogeneous materials with complex microstructures in an averaged or homogenized sense, various homogenization techniques are typically used (e.g. [59], [52], [39], [40], [47]). For numerical modelling of the heterogeneous materials, one straightforward approach is to mesh the whole structure, including all heterogeneities, which leads to giant computations. There have been some efforts to improve this technique and reduce the computational cost like Ladevèze et al. [26]. This approach is related to domain decomposition techniques, LATIN method [25] and parallel computations. One alternative is the multilevel finite element technique $\left(\mathrm{FE}^{2}\right)$ [15], which is based on multilevel computational procedures, and simply correlates the micro structural response with the overall macroscopic behaviour, irrespective of the deformation level or the material behaviour. The representative volume element (RVE) is introduced to decouple, in a computational sense, the macrostructure from the microstructure. Then the local microstructure is defined by a unique, discretised RVE in each integration point of the discretised homogenized macrostructure. The macroscopic stresses are obtained by direct averaging of the microscopic stresses, found by solving a nonlinear finite element problem on the representative volume element associated with the micro scale ([51], [15], [16], [53], [18], [60], [38]).

In most of these techniques, which are called first-order computational homogenization, the micro structural length scale is assumed infinitely small compared to the characteristic macro structural size. For problems where the micro structural length scale is comparable with the characteristic size of the macro component or with the length scale related to the macroscopic spatial variability in the loading, some authors proposed to use second-order in geometrical or constitutive relations, (see e.g. [23]). These techniques are named second-order computational homogenization.

The instability phenomena in the framework of multiscale homogenization analysis of heterogeneous materials are of structural and/or material types. These phenomena can occur at both the macro and micro scales and may influence each other. Abeyaratne and Triantafyllidis [1] have investigated for the first time the instability phenomena in the heterogeneous materials. They studied the macroscopic instability of a finitely strained periodic porous elastomer with a strictly rank-one convex matrix by homogenizing the tangent moduli. Moreover, the bifurcation of fiber-reinforced layered composite materials was investigated by Triantafyllidis and Maker [55]. A rigorous connection between bifurcation instability at the microscopic level and loss of rank-one convexity of the homogenized moduli in finitely strained periodic elastomers of infinite extent was shown by Geymonat et al. [17]. Triantafyllidis and Bar- 
denhagen [54] defined the onset-of-failure surfaces in stress and strain space for periodic solids of infinite extent, a concept which was subsequently applied among other solids to the case of trusses by Schraad and Triantafyllidis [50], biaxially compressed aluminium honeycomb by Triantafyllidis and Schraad [56], fiber-reinforced composites under combined normal and shear strains by Nestorović and Triantafyllidis [41], to three-dimensional Kelvin foams (Gong et al. [19]) and more recently to periodic porous and particulate elastomers by Triantafyllidis et al. [57]. Miehe et al. [36] have developed a general framework for the theoretical and computational treatment of these instability problems based on an investigation of the properties and the interaction of two coupled minimization principles: the principle of minimum potential energy of the macrostructure and the principle of minimum average energy of the microstructure. In addition, deBotton et al. [10] have derived an estimate for fiber-reinforced elastomers with incompressible Neo-Hookean phases and the special composite cylinder assemblage microstructure of Hashhin [21]. Furthermore, the second-order homogenization estimates have been used for studying the instability of heterogeneous materials ([29], [30], [31], [34], [32], [33]). Other attempts in this context of instability have been made by developing a framework based on a two-scale approach of the updated Lagrangien type ([42], [43], [44], [45], [13], [46]).

Generally, the resulting equations of the nonlinear formulation of the homogenization problem are solved using the classical Newton-Raphson procedure. The principle of this technique is to follow the solution branches in a stepwise manner via a succession of linearization and some iteration to achieve the equilibrium [8], [22]. It is well known that, for severe nonlinearities of the response curve with limit loading or displacement points, this technique requires elaborated procedure to overcome these difficulties [27]. This leads to very small step sizes and to increase the number of iterations to achieve the full computation procedure. This drawback becomes very important in presence of heterogeneous materials where the computation of localisation and homogenization tensors needs large consuming time.

In the present paper, we propose a numerical method to solve nonlinear problems in the framework of heterogeneous materials. This technique combines two modern tools: it uses first the multilevel finite element method $\left(F E^{2}\right)$ [15], [16] which was described before. The second tool is related to the numerical procedure to solve the resulting nonlinear problem. It is based on the asymptotic numerical method (ANM) [6], [7]. The ANM appears as an efficient continuation method without any corrector iteration. The principle of the ANM is to build up the solution branch in the form of power series that are truncated at rather large orders. With such a representation, one gets approximations of the solution path that are very accurate inside the radius of convergence. Introducing such expansion in the nonlinear problems at both macro and micro scales, it results a series of linear problems that must be solved successively. Compared to the work presented in Feyel and Chaboche [15] which combine $\mathrm{FE}^{2}$ method and Newton-Raphson algorithm, our technique, 
named Multiscale-ANM, offers the following advantages: (i) in the context of a multilevel finite element analysis, the solutions of the nonlinear microscopic problems are obtained by solving series of linear problems. One straightforward consequence is that a localization tensor can then be constructed for each step of the asymptotic development and an explicit constitutive law can then be constructed at the macroscopic level at each step. Thus, while in the $\mathrm{FE}^{2}$ method the linearization is only valid at each Newton-Raphson iteration, in our procedure the linearization remains valid along the whole asymptotic step; (ii) in the ANM, the response curve is explicitly represented in the form of a vectorial series which is calculated with only one decomposition of the tangent matrix. This representation with series contains several informations that are inaccessible by the classical algorithms. Furthermore, the length of each step of calculation is a posteriori estimated by analysing the computed terms of the series and which is often much larger than the one imposed in Newton-Raphson procedure (i.e. the response is obtained with fewer steps and, consequently the computational cost is drastically reduced); (iii) compared to Newton-Raphson-based methods, ANM is a very simple technique for solving problems involving junctions and instabilities thanks to its high order approximation [64], [3], [61].

The layout of this paper is as follows: in section 2, the formulation of micromacro problem is described. In section 3, details of the perturbation procedure applied to the multiscale problem are given. In section 4 , the technique is first validated by a two scale problem on a nonlinear heterogeneous structure. Two examples are then presented, where instabilities at the local level introduce a significant modification of the macroscopic structure response.

\section{Problem formulation}

\subsection{Problem at the macroscopic scale}

We consider a domain $\Omega$ in $\mathbb{R}^{D}, D$ being the domain dimension, with an external boundary $\partial \Omega$, describing the structure at the macroscopic level in its reference configuration. We assume that the material is heterogeneous and characterized by a periodic microstructure. The structure is subjected to prescribed displacements and forces on the disjoint complementary parts of the boundary $\partial \Omega_{u}$ (the Dirichlet boundaries) and $\partial \Omega_{F}$ (the Neumann boundaries). Large strains are considered and a total Lagrangian formulation is adopted. In the following, the notation (.) will be used to denote macroscopic quantities. The problem to solve is defined as follows, in the absence of body forces:

$$
\nabla \cdot \overline{\mathbf{P}}(\overline{\mathbf{X}})=0 \quad \text { in } \Omega
$$


where $\overline{\mathbf{P}}(\overline{\mathbf{X}})$ is the first Piola-Kirchhoff tensor associated with a point $\overline{\mathbf{X}}$ of the macroscopic structure in its initial configuration. The boundary conditions are defined by:

$$
\left\{\begin{array}{lll}
\overline{\mathbf{u}}(\overline{\mathbf{X}})=\hat{\mathbf{u}}(\overline{\mathbf{X}}) & \text { on } & \partial \Omega_{u} \\
\overline{\mathbf{P}} \mathbf{N}=\lambda \mathbf{f} & \text { on } & \partial \Omega_{F}
\end{array}\right.
$$

where $\overline{\mathbf{u}}=\overline{\mathbf{x}}-\overline{\mathbf{X}}$ denotes the macroscopic displacement field, $\overline{\mathbf{x}}$ being the coordinates of a point in the deformed configuration, and $\hat{\mathbf{u}}$ are the prescribed displacements. In Eq. (2), $\mathbf{N}$ is the outward unit normal vector to $\partial \Omega, \mathbf{f}$ is a prescribed load and $\lambda$ is a parameter that gives the intensity of the applied load.

The weak form associated with equations (1)-(2) is given by:

Find $\overline{\mathbf{u}} \in \mathcal{S}(\Omega), \overline{\mathbf{u}}=\hat{\mathbf{u}}$ on $\partial \Omega_{u}$ such that:

$$
\int_{\Omega} \overline{\mathbf{P}}: \nabla_{\bar{X}} \boldsymbol{\delta} \overline{\mathbf{u}} d \Omega=\int_{\Omega} \overline{\mathbf{P}}: \boldsymbol{\delta} \overline{\mathbf{F}} d \Omega=\lambda \int_{\partial \Omega_{F}} \mathbf{f} \cdot \boldsymbol{\delta} \overline{\mathbf{u}} d \Gamma \quad \forall \boldsymbol{\delta} \overline{\mathbf{u}} \in \mathcal{S}_{0}(\Omega)
$$

where $\mathcal{S}(\Omega)$ denotes the space of sufficiently regular displacements and $\mathcal{S}_{0}(\Omega)=$ $\left\{\boldsymbol{\delta} \overline{\mathbf{u}} \in \mathcal{S}(\Omega), \boldsymbol{\delta} \overline{\mathbf{u}}=0\right.$ on $\left.\partial \Omega_{u}\right\}$ is the corresponding space of suitable variations with vanishing values on the Dirichlet boundary. Let $\overline{\mathbf{F}}=\boldsymbol{\nabla}_{\bar{X}} \overline{\mathbf{u}}+\mathbf{I}$ the macroscopic deformation gradient tensor, I being the second-order identity tensor. At this scale, the constitutive relationship between $\overline{\mathbf{P}}$ and $\overline{\mathbf{F}}$ is unknown. In the context of multilevel finite element analysis, the macroscopic stresses are extracted directly by solving a local nonlinear finite element problem related with the periodic microstructure. The associated formulation is presented in the next section.

\subsection{Problem at the microscopic scale}

We assume that the material is heterogeneous with a periodic microstructure, characterized by a representative volume element (RVE) that occupies a domain $\omega$ in $\mathbb{R}^{D}$ with external boundary $\partial \omega$ in its reference configuration. The equilibrium equation is given in the absence of body forces by:

$$
\boldsymbol{\nabla} \cdot \mathbf{P}(\mathbf{X})=0 \quad \text { in } \omega
$$

where $\mathbf{P}(\mathbf{X})$ is the first Piola-Kirchhoff stress tensor at a microscopic point $\mathbf{X}$. The RVE is subjected to boundary conditions (b.c.) depending on the macroscopic deformation tensor $\overline{\mathbf{F}}$. Different types of b.c. can be chosen such as linear deformations, uniform tractions, or periodic constraints (see e.g. [37]). We consider in this work linear deformations (5) and periodic conditions (6) 
on the boundary of the RVE:

$$
\begin{aligned}
& \mathbf{u}=(\overline{\mathbf{F}}-\mathbf{I}) \mathbf{X} \text { on } \partial \omega, \\
& \mathbf{u}^{+}-\mathbf{u}^{-}=(\overline{\mathbf{F}}-\mathbf{I})\left(\mathbf{X}^{+}-\mathbf{X}^{-}\right) \text {on } \partial \omega,
\end{aligned}
$$

where $\mathbf{u}$ is the microscopic displacement. In Eq. (6), the exponents + and - are associated with node indices on opposite sides of the RVE. At the microscopic scale, we assume that the constitutive relations are known in each phase of the RVE. We consider large displacement model and a linear constitutive relation for each phase:

$$
\mathbf{S}(\mathbf{X})=\mathbb{C}^{(r)}: \mathbf{E}(\mathbf{X})
$$

where $\mathbf{S}$ denotes the second Piola-Kirchhoff stress tensor related to $\mathbf{P}$ through $\mathbf{P}=\mathbf{F S}$, where $\mathbf{F}$ is the microscopic deformation gradient tensor, $\mathbb{C}^{(r)}$ and E refer to fourth-order elastic tensor associated with phase $(r)$, and GreenLagrange strain tensor, respectively. It is worth noting that any other constitutive law (including inelastic behaviours) can be used in the context of the asymptotic numerical method (see examples in viscoplasticity and plasticity in previous ANM papers ([11], [62], [63]). The weak form associated with the microscopic problem (4) is written as:

Find $\mathbf{u} \in \mathcal{S}(\omega)$ and satisfying microscopic Dirichlet boundary conditions (equation (5) or (6)), such that:

$$
\int_{\omega} \mathbf{P}: \nabla_{X} \boldsymbol{\delta} \mathbf{u} d \omega=\int_{\omega} \mathbf{S}: \delta \mathbf{E} d \omega=0 \quad \forall \boldsymbol{\delta} \mathbf{u} \in \mathcal{S}_{0}(\omega)
$$

The two problems at the micro and macro scales are coupled through the following relation, that enables to determine the effective stress $\overline{\mathbf{P}}$ at a particular point $\overline{\mathbf{X}}$ of the macroscopic domain $\Omega$ where the stress state is assumed to be homogeneous in the neighbourhood of $\overline{\mathbf{X}}$ :

$$
\overline{\mathbf{P}}=\langle\mathbf{P}(\mathbf{X})\rangle=\frac{1}{|\omega|} \int_{\omega} \mathbf{P}(\mathbf{X}) d \omega,
$$

where $|\omega|$ is the volume of the RVE. Note that the macroscopic gradient is also the mean value of the microscopic deformation gradient:

$$
\overline{\mathbf{F}}=\langle\mathbf{F}(\mathbf{X})\rangle=\frac{1}{|\omega|} \int_{\omega} \mathbf{F}(\mathbf{X}) d \omega
$$

The relation (10) follows from the boudary conditions (5) and (6) imposed on the RVE (see e.g. [37]). 


\section{Asymptotic formulation of the multiscale problem}

Solution of the nonlinear multiscale problems (3), (5), (6), (7), (8) and (9) is sought using the asymptotic numerical method. It consists in expanding the main variables of the problem into power series with respect to a path parameter. In this way, the nonlinear problem is transformed into a sequence of linear problems having the same tangent operator. By comparison with Newton-Raphson procedure, ANM can be considered as a high order predictor without need of any iteration. Many applications of ANM to structural and fluid mechanics show the performance of this technique with respect to computation time, adaptability of the step length and the treatment of instabilities. For the problem considered in the proposed study, both variables of macroscale and microscale are expanded into power series. We propose to represent these variables in a mixed vector $\Lambda=(\overline{\mathbf{u}}, \overline{\mathbf{P}}, \mathbf{u}, \mathbf{P}, \ldots)$. The perturbation technique is applied to $\Lambda$ and to the load parameter $\lambda$, leading to the following expression:

$$
\left\{\begin{array}{l}
\boldsymbol{\Lambda}(a) \\
\lambda(a)
\end{array}\right\}=\left\{\begin{array}{c}
\boldsymbol{\Lambda}_{0} \\
\lambda_{0}
\end{array}\right\}+\sum_{p=1}^{N} a^{p}\left\{\begin{array}{c}
\boldsymbol{\Lambda}_{p} \\
\lambda_{p}
\end{array}\right\}
$$

where $().(a)$ refers to quantities defined continuously with respect to the scalar parameter $a,(.)_{0}$ denotes a known initial solution such that $().(0)=(.)_{0}, N$ is the truncation order of the series, and $(.)_{p}$ indicates a term at order $p$ that has to be determined. To solve the resulting linear problems, we need to define the parameter $a$ to carry out the computation in the same way as in the classical iterative algorithms in which the computation can be managed by imposing a force, a displacement or a combination of both (i.e. Riks technique [49]). In the proposed algorithm, we have chosen the projection of the macroscopic displacement field onto its tangent leading to:

$$
a=\left(\overline{\mathbf{u}}(a)-\overline{\mathbf{u}}_{0}\right) \cdot \overline{\mathbf{u}}_{1}
$$

Because the series has a convergence radius, a simple procedure allows one to determine a validity range of the truncated series, which gives the maximum value of the parameter $a$ noted $a_{\max }$ :

$$
a_{\max }=\left(\delta \frac{\left\|\overline{\mathbf{u}}_{1}\right\|}{\left\|\overline{\mathbf{u}}_{N}\right\|}\right)^{\frac{1}{N-1}} .
$$

where $\delta$ is the accuracy parameter and $\|$. $\|$ refers to the norm of variables. In this way, the whole solution is obtained in a step by step manner considering each end step as the starting point for the next one. Details of these procedures in ANM framework can be consulted in the references ([6], [64], [4], [7]). 


\subsection{Asymptotic expansion at the macroscopic problem}

The nonlinear problem (3) is expanded by using (11):

$$
\int_{\Omega}\left(\overline{\mathbf{P}}_{0}+\sum_{p=1}^{N} a^{p} \overline{\mathbf{P}}_{p}\right): \boldsymbol{\delta} \overline{\mathbf{F}} d \Omega=\left(\lambda_{0}+\sum_{p=1}^{N} a^{p} \lambda_{p}\right) \int_{\partial \Omega_{F}} \mathbf{f} \cdot \boldsymbol{\delta} \overline{\mathbf{u}} d \Gamma .
$$

Collecting terms with the same powers of $a$ induces a sequence of linear problems defined as follows:

$$
\int_{\Omega} \overline{\mathbf{P}}_{p}: \delta \overline{\mathbf{F}} d \Omega=\lambda_{p} \int_{\partial \Omega_{F}} \mathbf{f} \cdot \boldsymbol{\delta} \overline{\mathbf{u}} d \Gamma \quad \forall p=1, \ldots, N .
$$

If the macroscopic constitutive law is known, each problem at order $p$ can be solved for $\mathbf{u}_{p}$ and $\lambda_{p}$ using the previous solutions of order $i<p$. As both variables $\mathbf{u}_{p}$ and $\lambda_{p}$ are unknown, one additional equation to (15) is needed. For this purpose, expression (12) is expanded using (11) which gives an additional equation at each order $p$. A complete detailed description of the procedure to solve these problems can be found in previous cited references about ANM. In our case, since the macroscopic constitutive relation is unknown, it is needed to carry out a computation on the microscopic problem to extract it.

\subsection{Asymptotic expansion at the microscopic problem}

The microscopic problem is defined as follows:

$$
\left\{\begin{array}{l}
\int_{\omega} \mathbf{S}: \boldsymbol{\delta} \mathbf{E} d \omega=0 \text { in } \omega \\
\mathbf{u}=(\overline{\mathbf{F}}-\mathbf{I}) \mathbf{X} \text { or } \mathbf{u}^{+}-\mathbf{u}^{-}=(\overline{\mathbf{F}}-\mathbf{I})\left(\mathbf{X}^{+}-\mathbf{X}^{-}\right) \text {on } \partial \omega
\end{array}\right.
$$

where

$$
\begin{aligned}
& \mathbf{S}=\mathbb{C}^{(r)}: \mathbf{E}, \\
& \mathbf{E}=\frac{1}{2}\left(\mathbf{F}^{T} \mathbf{F}-\mathbf{1}\right)=\frac{1}{2}\left(\boldsymbol{\nabla}_{\mathbf{X}} \mathbf{u}+\boldsymbol{\nabla}_{\mathbf{X}} \mathbf{u}^{T}+\boldsymbol{\nabla}_{\mathbf{X}} \mathbf{u}^{T} \boldsymbol{\nabla}_{\mathbf{X}} \mathbf{u}\right) .
\end{aligned}
$$

The ANM expansion of equations (16) leads to a sequence of linear problems which can be expressed in the following form at order $p(1 \leq p \leq N)$ :

$$
\begin{aligned}
& \mathcal{L}\left(\mathbf{u}_{p}, \boldsymbol{\delta} \mathbf{u}\right)=\mathcal{F}_{p}^{n l}(\boldsymbol{\delta} \mathbf{u}), \\
& \mathbf{u}_{p}=\overline{\mathbf{F}}_{p} \mathbf{X} \text { or } \mathbf{u}_{p}^{+}-\mathbf{u}_{p}^{-}=\overline{\mathbf{F}}_{p}\left(\mathbf{X}^{+}-\mathbf{X}^{-}\right) \text {on } \partial \omega .
\end{aligned}
$$


The explicit form of the operators $\mathcal{L}$ and $\mathcal{F}_{p}^{n l}$ is given in Appendix I. It is worth noting that the tangent opertator $\mathcal{L}$ is well defined and is the same for all orders of the series. The right-hand side term of equation $(18), \mathcal{F}_{p}^{n l}(\boldsymbol{\delta} \mathbf{u})$, is calculated from the obtained solutions of the previous orders $p>1$ and equal to zero for $p=1$. Note that the boundary conditions (19) depend on the local macroscopic gradient deformation, $\overline{\mathbf{F}}_{p}$, which is unknown.

As (18) are linear problems, we can apply the superposition principle. Therefore, the solution at order $p, \mathbf{u}_{p}$, can be expressed as a linear combination of the solution modes obtained by imposing independently the homogeneous deformation fields through the boundary conditions (19) (see Appendix II). At order $p$ the solution $\mathbf{u}_{p}$ can then be expressed as:

$$
\mathbf{u}_{p}(\mathbf{X})=\mathbb{A}(\mathbf{X}): \overline{\mathbf{F}}_{p}+\mathbf{u}_{p}^{n l}(\mathbf{X})
$$

where $\mathbb{A}$ is a third-order tensor. In equation (20), $\mathbf{u}_{p}^{n l}$ is a vector associated with the right-hand side term in equation (18). In the following, we omit the $\mathbf{X}$ dependence for the sake of clarity when no confusion is possible. Expanding the gradient deformation tensor $\mathbf{F}$ it results:

$$
\mathbf{F}=\mathbf{I}+\boldsymbol{\nabla}_{\mathbf{X}} \mathbf{u}=\mathbf{I}+\boldsymbol{\nabla}_{\mathbf{X}} \mathbf{u}_{0}+\sum_{p=1}^{N} a^{p} \boldsymbol{\nabla}_{\mathbf{X}} \mathbf{u}_{p} .
$$

Deriving (20) with respect to $\mathbf{X}$ and using $\boldsymbol{\nabla}_{\mathbf{X}} \mathbf{u}_{p}=\mathbf{F}_{p}$, we obtain:

$$
\mathbf{F}_{p}=\mathbb{A}_{, \mathbf{X}}: \overline{\mathbf{F}}_{p}+\mathbf{u}_{p, \mathbf{X}}^{n l}
$$

where $\mathbb{A}_{, \mathbf{X}}$ is a fourth-order tensor which is identified as a localization tensor, and $\mathbf{u}_{p, \mathbf{X}}^{n l}$ is a second-order tensor.

To achieve the homogenized relationship, we consider the asymptotic expansion of $\mathbf{P}=\mathbf{F S}$. After some elementary operations, one obtains at order $p$ the following relation :

$$
\mathbf{P}_{p}=\mathbb{L}: \overline{\mathbf{F}}_{p}+\mathbf{P}_{p}^{n l}
$$

where expressions of operators $\mathbb{L}$ and $\mathbf{P}_{p}^{n l}$ are detailed in Appendix III. The effective stresses can then be obtained at each order $p$ by averaging equation (23):

$$
\overline{\mathbf{P}}_{p}=\overline{\mathbb{L}}: \overline{\mathbf{F}}_{p}+\overline{\mathbf{P}}_{p}^{n l}
$$

with (see Appendix III)

$$
\overline{\mathbb{L}}=\left\langle\mathbb{H}^{(r)}: \mathbb{A}_{, X}\right\rangle=\langle\mathbb{L}\rangle, \quad \overline{\mathbf{P}}_{p}^{n l}=\left\langle\mathbf{P}_{p}^{n l}\right\rangle
$$


Box 1. Summary of the homogenization procedure at order $p$.

At order $p$ :

(i) : Expand $\mathbf{u}_{p}$ as a linear form of $\overline{\mathbf{F}}_{p}$ (Appendix II and equation (20)) :

$\mathbf{u}_{p}(\mathbf{X})=\mathbb{A}(\mathbf{X}): \overline{\mathbf{F}}_{p}+\mathbf{u}_{p}^{n l}(\mathbf{X})$

(ii) : Construct the localization tensor $\mathbb{A}_{, \mathbf{X}}$ (equations (21-22)) :

$\mathbf{F}_{p}(\mathbf{X})=\mathbb{A}_{, \mathbf{X}}(\mathbf{X}): \overline{\mathbf{F}}_{p}+\mathbf{u}_{p, \mathbf{X}}^{n l}(\mathbf{X})$

(iii) : Express $\mathbf{P}_{p}$ using the asymptotic expansion of $\mathbf{P}(\mathbf{X})=\mathbf{F}(\mathbf{X}) \mathbf{S}(\mathbf{X})$ and the known constitutive relationship at the micro level (Appendix III and equation

$(23))$ :

$\mathbf{P}_{p}(\mathbf{X})=\mathbb{L}(\mathbf{X}): \overline{\mathbf{F}}_{p}+\mathbf{P}_{p}^{n l}(\mathbf{X})$

(iv) : Average to find the effective constitutive law at order $p$ :

$\overline{\mathbf{P}}_{p}=\overline{\mathbb{L}}: \overline{\mathbf{F}}_{p}+\overline{\mathbf{P}}_{p}^{n l}$

We can note a direct analogy with linear homogenization, where $\mathbb{H}^{(r)}$ replaces the linear elastic tensor of phase $(r)$ and $\mathbb{A}_{, X}$ replaces the localization tensor relating the linearized microscopic strains $\boldsymbol{\epsilon}(\mathbf{y})$ to the linearized macroscopic strains $\overline{\boldsymbol{\epsilon}}, \mathbf{y}$ denoting the microscopic coordinates.

Introducing (25) in equation (15), we obtain the final form of the linear problem at order $p$ at the macroscopic level:

$$
\int_{\Omega} \overline{\mathbf{F}}_{p}: \overline{\mathbb{L}}: \delta \overline{\mathbf{F}} d \Omega=\lambda_{p} \int_{\partial \Omega_{F}} \mathbf{f} \cdot \boldsymbol{\delta} \mathbf{u} d \Gamma-\int_{\Omega} \overline{\mathbf{P}}_{p}^{n l}: \boldsymbol{\delta} \overline{\mathbf{F}} d \Omega
$$

The layout of the homogenization procedure is summarized in Box 1. It is based on the property that each problem at order $p$ is linear. Then all the quantities, including $\mathbf{u}_{p}, \overline{\mathbf{F}}_{p}, \mathbf{F}_{p}$ and $\mathbf{P}_{p}$ can be related linearly, and extracting the constitutive law is possible by constructing a localization tensor at order $p$.

In conclusion, we have shown that using an asymptotic expansion at both micro and macro levels transforms the multilevel nonlinear problem into a sequence of linear problems. At each order $p$, the superposition principle can then be used to construct the localization tensor and then an explicit expression for the constitutive effective law. The second advantage of this formulation is, as it has been shown in previous papers about ANM, that instabilities and post-buckling problems can be treated without the issues associated with Newton-Raphson procedures that break down in presence of instabilities, and require special arc-length techniques. Furthermore, as the solution is in the present context described continuously with respect to the development parameter $a$, large steps can be obtained, that lead to an efficient numerical procedure to describe multiscale buckling. In the following, the weak forms 
(26) and (18) are discretized and solved using the finite element method.

\section{$4 \quad$ Numerical examples}

We study the accuracy and the efficiency of the proposed technique through several nonlinear problems. The results are presented here through two main examples. The first one concerns the bending of a rectangular beam made of a heterogeneous material. It is used to show validity of the algorithm associating $\mathrm{ANM}$ and $\mathrm{FE}^{2}$, which is presented here for the first time. It is then required to be very simple, and does not involve any local instability. The proposed approach is validated by comparing it with a fully meshed model. In this latter, details of all heterogeneities are meshed. The second example deals with the compression of a composite material where local instabilities induce a modification of the macroscopic structure response. The proposed problems have been discretized using two-dimensional finite element in the plane stress framework. The macrostructures have been meshed with four node quadrangular elements whereas the microstructures have been meshed with three node triangular elements or nine node quadrangular elements.

In the ANM procedure, two main parameters are important: the truncation order $\mathrm{N}$ of the series which is usually chosen between 10 and 20, and the accuracy parameter $\delta$ which allows limiting the length of each asymptotic step in a continuation procedure. In all the tests presented here, we have solved the nonlinear problems using ANM with $N=15$ and $\delta=10^{-6}$. (For more information, we refer to [27], [2], [3], [61], [7]).

We denote by $V$ the RVE volume, $E_{i}, \nu_{i}$ and $V_{i}$ are the Young modulus, the Poisson's ratio and of the matrix for $i=1$ and volume of the inclusion for $i=2$, respectively. For all the examples, the Poisson's ratio is equal to 0.3.

\subsection{Bending of a rectangular beam}

In this example, we consider the bending of a heterogeneous rectangular beam. The specimen is submitted to a concentrated load at the point $C$ and is clamped at its left edge as shown in figure 1 . We consider a linear deformation on the boundary of RVE as described in equation (5). This test is selected to assess the validity of the multiscale-ANM approach by studying different aspects involved in the homogenization technique. For this goal, three types of RVE are examined: (i) a biphasic RVE with circular inclusion (figure 2a), (ii) a biphasic RVE with vertical elliptical inclusion (figure 2b) and (iii) a biphasic RVE with horizontal elliptical inclusion (figure 2c). Furthermore, the influence of the rigidity ratios $E_{2} / E_{1}$ and of volume fractions $V_{2} / V$ are studied. We have compared results obtained with our multiscale procedure to those of a fully 


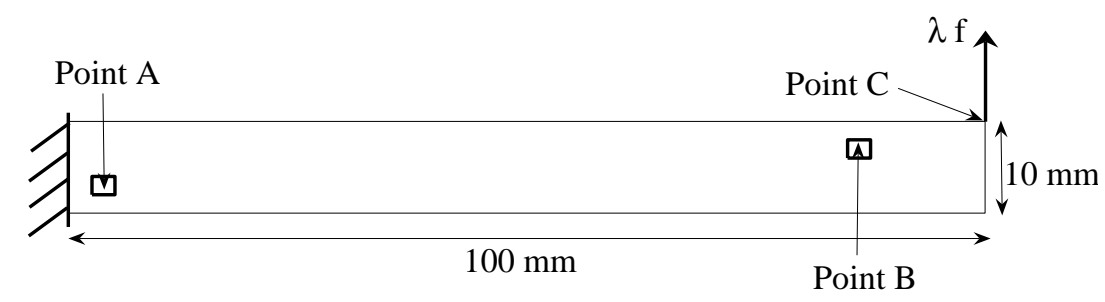

Fig. 1. Geometry of the bending problem and the cells considered to compare the stresses, $\mathrm{f}=1 \mathrm{~N}$

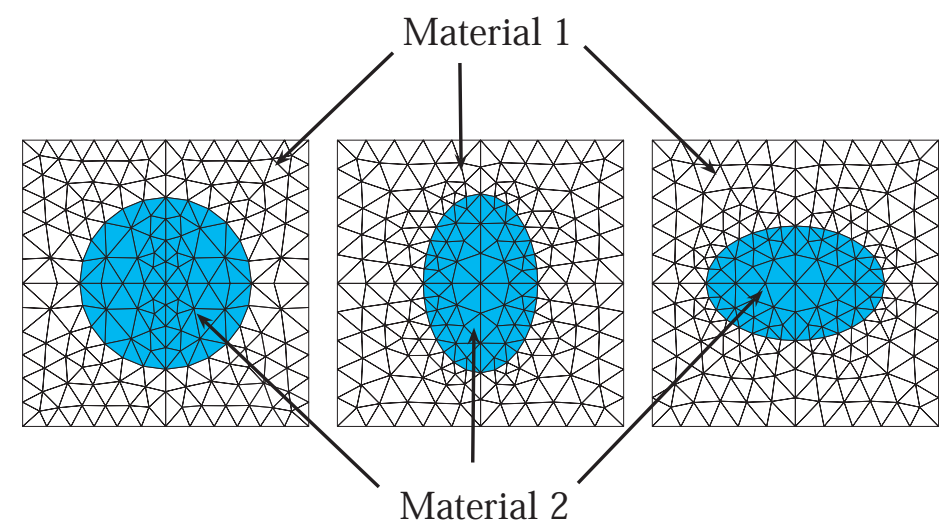

(a)

(b)

(c)

Fig. 2. (a) Biphasic RVE with circular inclusion, (b) biphasic RVE with vertical elliptical inclusion, and (c) biphasic RVE with horizontal elliptical inclusion.

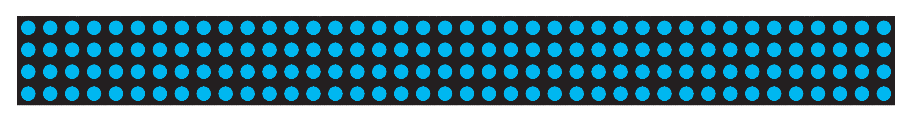

Fig. 3. Rectangular beam fully meshed model (58658 degrees of freedom)

meshed structure involving the explicit mesh of all heterogeneities (see figure $3)$.

To investigate the influence of the rigidity ratio, the biphasic heterogeneous materials with circular inclusion is considered. We have tested two different rigidity ratios $\left(E_{2} / E_{1}=10\right.$ and $\left.E_{2} / E_{1}=0.1\right)$. These correspond to two cases: first the inclusion is more rigid than the matrix $\left(E_{2} / E_{1}=10\right.$ and $E_{2}=100000 \mathrm{MPa}$ ), and second, the inclusion is less rigid than the matrix $\left(E_{2} / E_{1}=0.1\right.$ and $\left.E_{2}=10000 M P a\right)$. Figure 4 shows the displacements of the point $C$ versus the loading parameter $\lambda$ for the two approaches (multiscaleANM and fully meshed model). The squares and circles indicate the end of the asymptotic steps. We note that the proposed multiscale procedure can reproduce with very good accuracy the complete detailed heterogeneous structure response. Figure 5 shows the deformed mesh of the equivalent homogeneous macro structure. This configuration is obtained with only two asymptotic steps using Multiscale-ANM approach. We also give the deformed microstructure for 


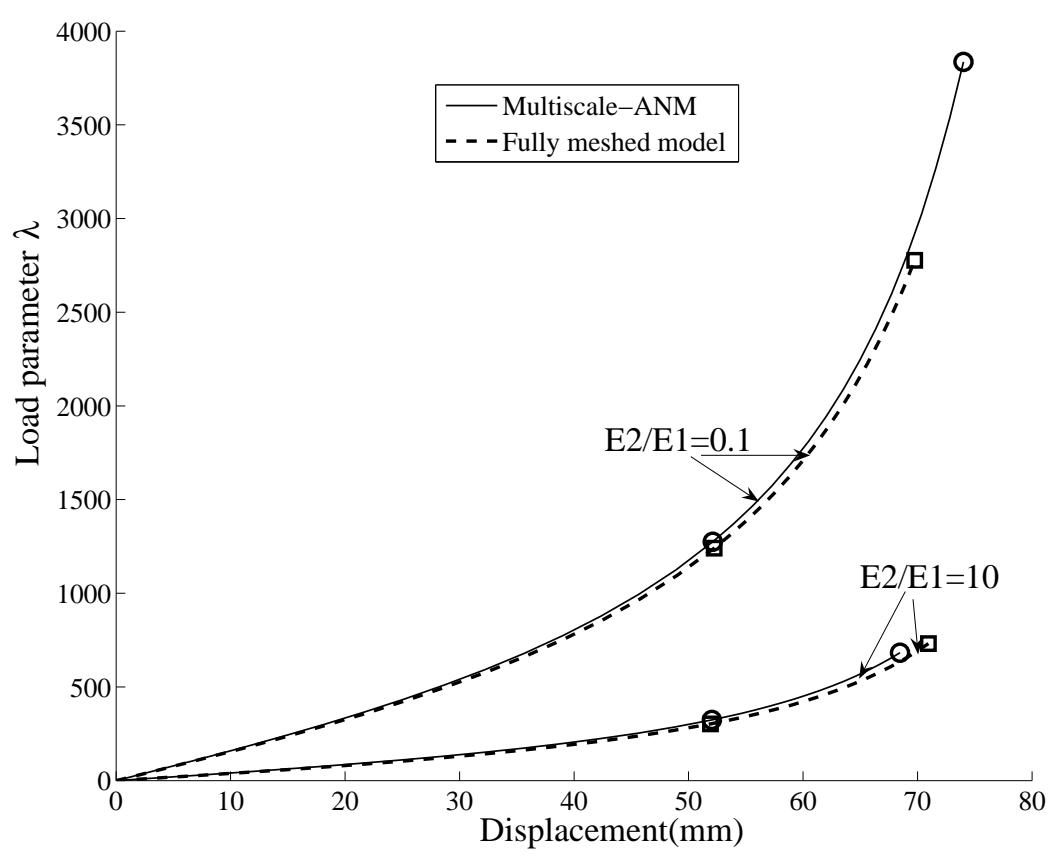

Fig. 4. The load-displacement diagrams of the bending problem for the biphasic RVE with circular inclusion and different rigidity ratios, displacement of point $C$.

two integration points at two different elements located at point $A$ and $B$ (see figure 1).

To study the influence of volume fraction, we consider the biphasic heterogeneous materials with circular inclusion, rigidity ratio of 10 and different volume fractions $\left(V_{2} / V=28 \%\right.$ and $\left.V_{2} / V=7 \%\right)$. In the figure (6), the displacements of the applied force point versus the loading parameter is shown. To analyse local variables obtained with the two approaches, the spatial averages of macro stresses at two cells located at points $A$ and $B$ have been calculated in the fully meshed model and compared with those obtained in the multiscale-ANM approach. Figures 7 and 8 show the averaged stresses $S_{11}, S_{22}$ and $S_{12}$ versus the loading parameter for the two models.

Finally, the biphasic heterogeneous materials with vertical and horizontal elliptical inclusions are considered. The rigidity ratio is equal to 10 and the volume fractions of two types of RVE are the same and equal to $20 \%$. The displacements of applied force versus the loading parameter are shown in figure 9 .

The proposed tests show the validity of the Multiscale-ANM approach since the results of this approach are quite similar to those of fully meshed model in all the tests. Note that with only two asymptotic steps, one obtains a vertical displacement of $70 \%$ of the beam length. Only two decompositions of the tangent stiffness matrix are needed which reduces significantly the computation time of micro-to-macro transition by comparison with the classical iterative Newton-Raphson procedure. This latter needs several steps and iterations to 


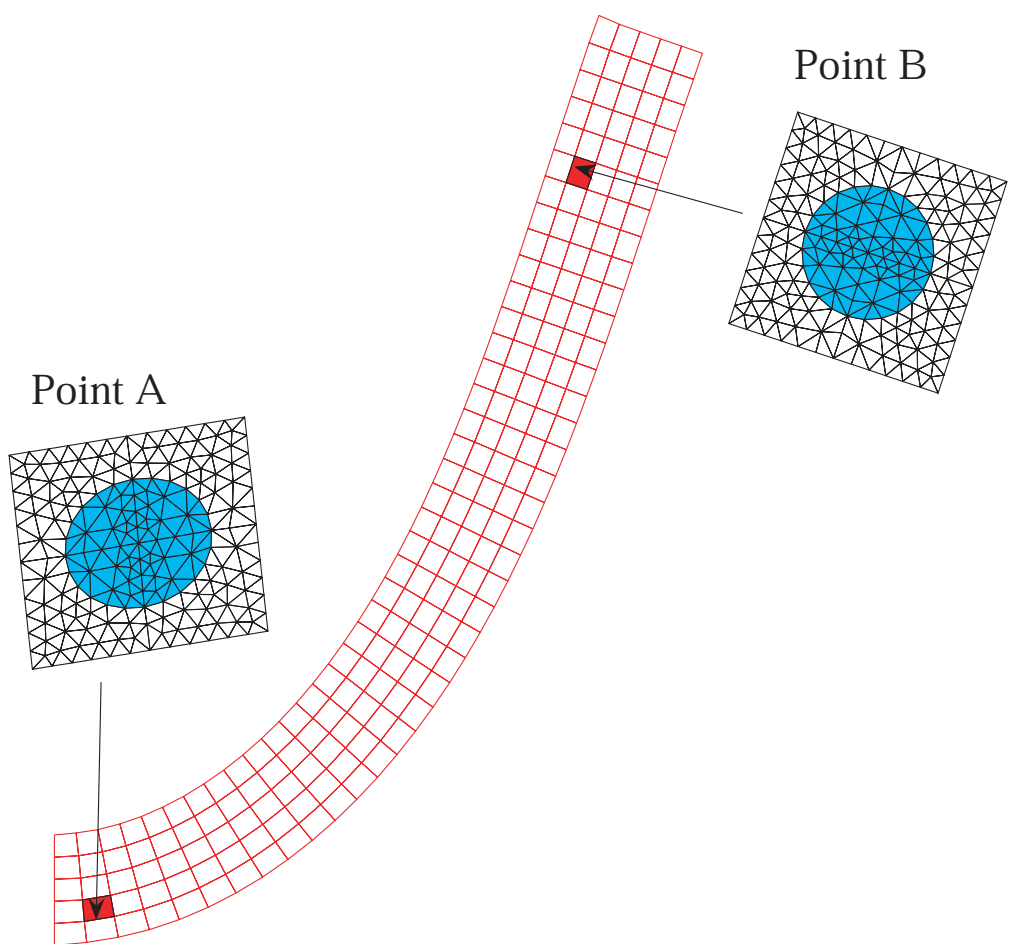

Fig. 5. Deformed shape of the structure and deformed microstructure at integration points after two asymptotic steps of Multiscale-ANM (Case considered: circular inclusion, inclusion volume fraction is $28 \%$ and rigidity ratios is 0.1 ).

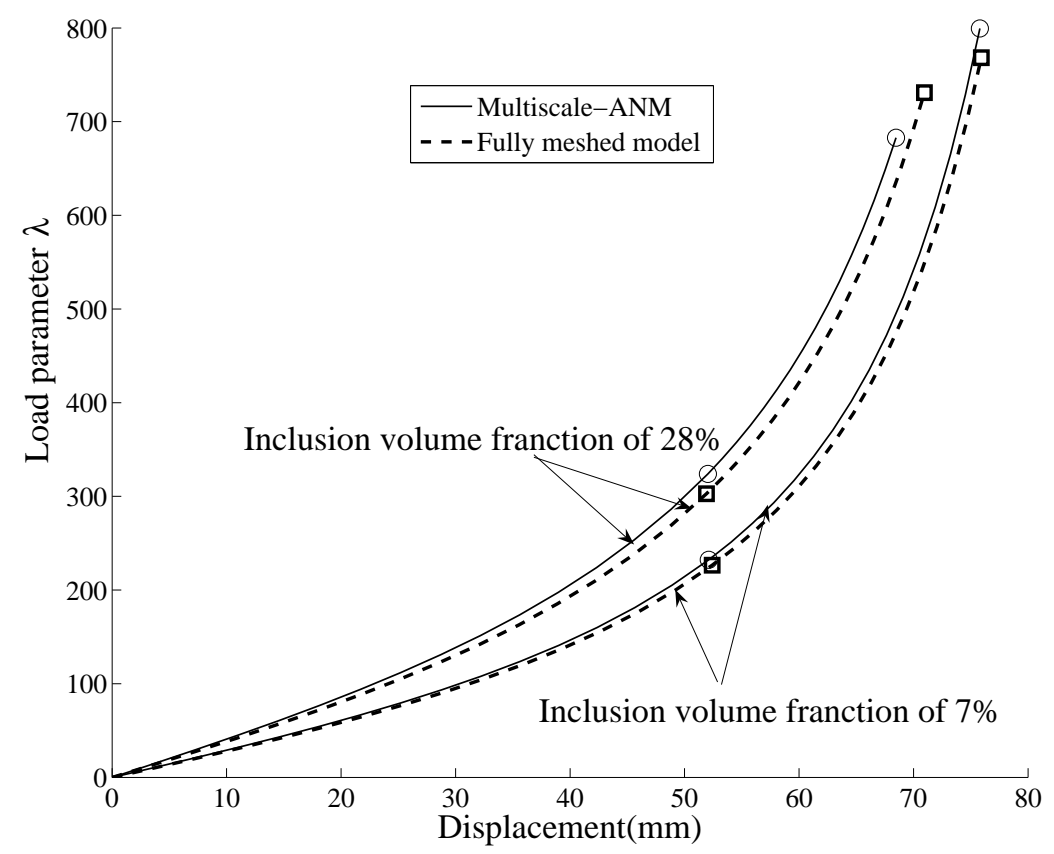

Fig. 6. The load-displacement diagrams for the biphasic RVE with circular inclusion, different volume fractions and rigidity ratio of 10 , displacement of point $C$. 


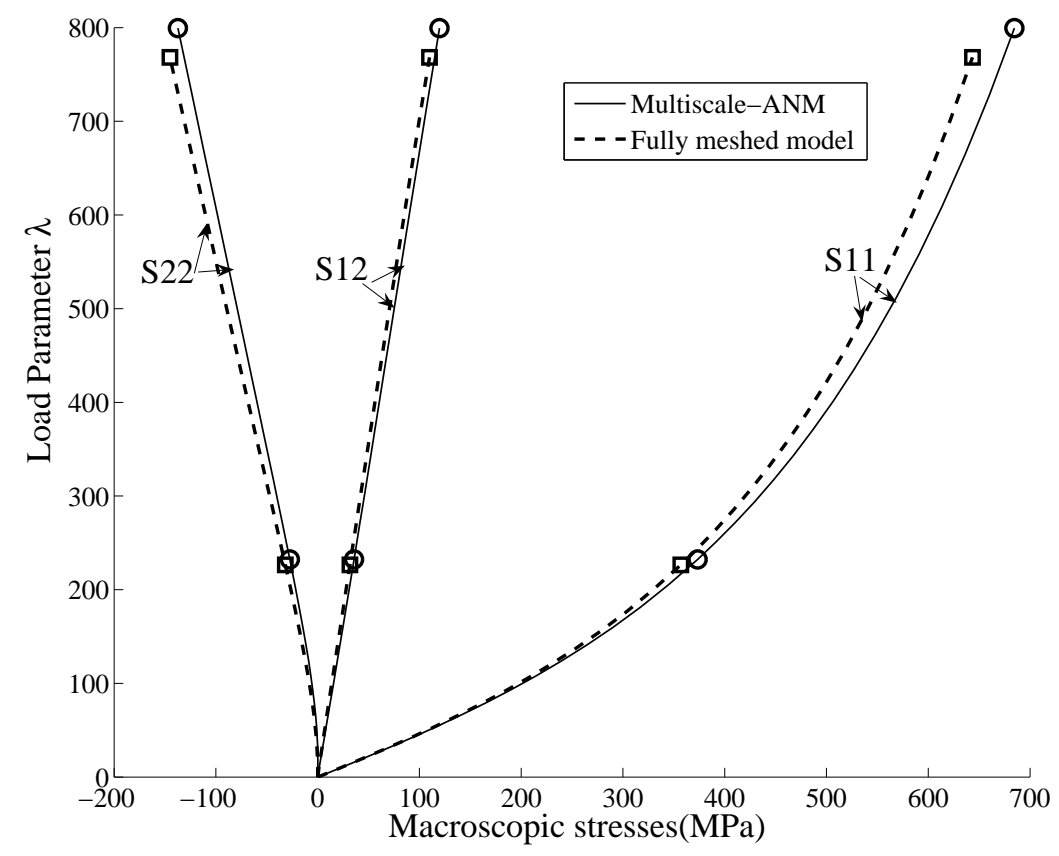

Fig. 7. The load-stress diagrams of the bending problem for the biphasic RVE with circular inclusion, volume fraction of $7 \%$ and rigidity ratio of 10 at the point A.

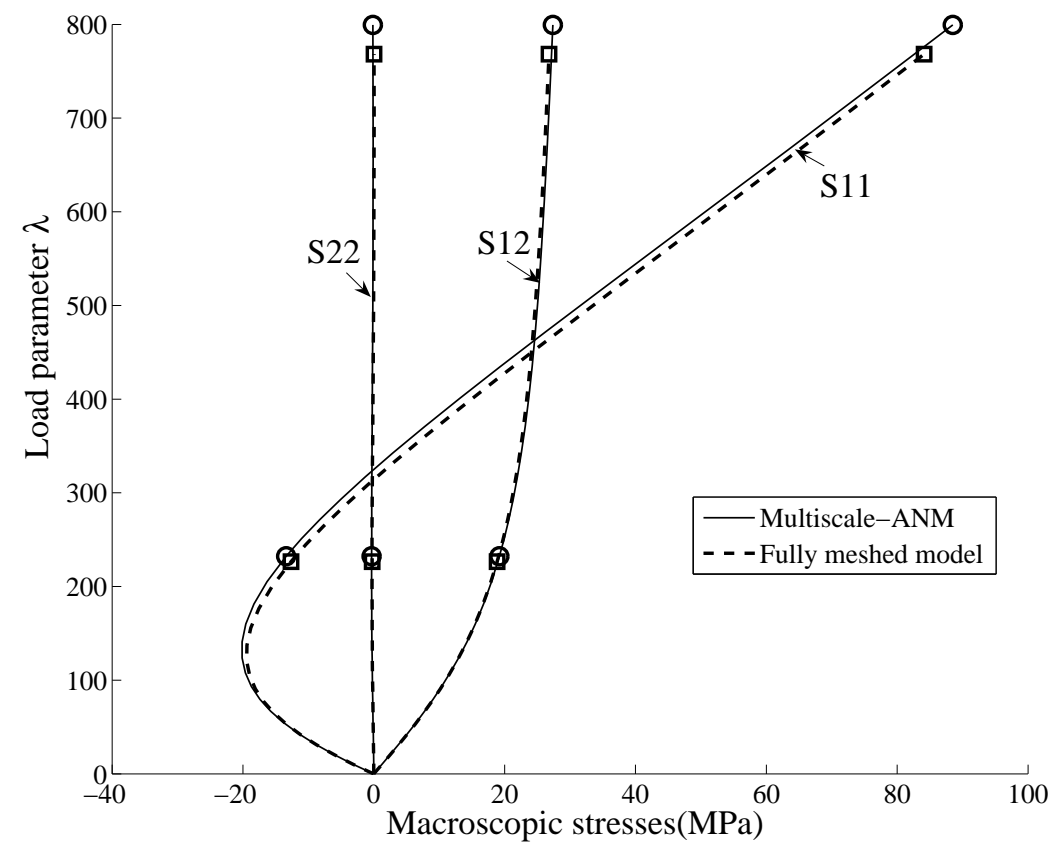

Fig. 8. The load-stress diagrams of the bending problem for the biphasic RVE with circular inclusion, volume fraction of $7 \%$ and rigidity ratio of 10 at the point $\mathrm{B}$. 


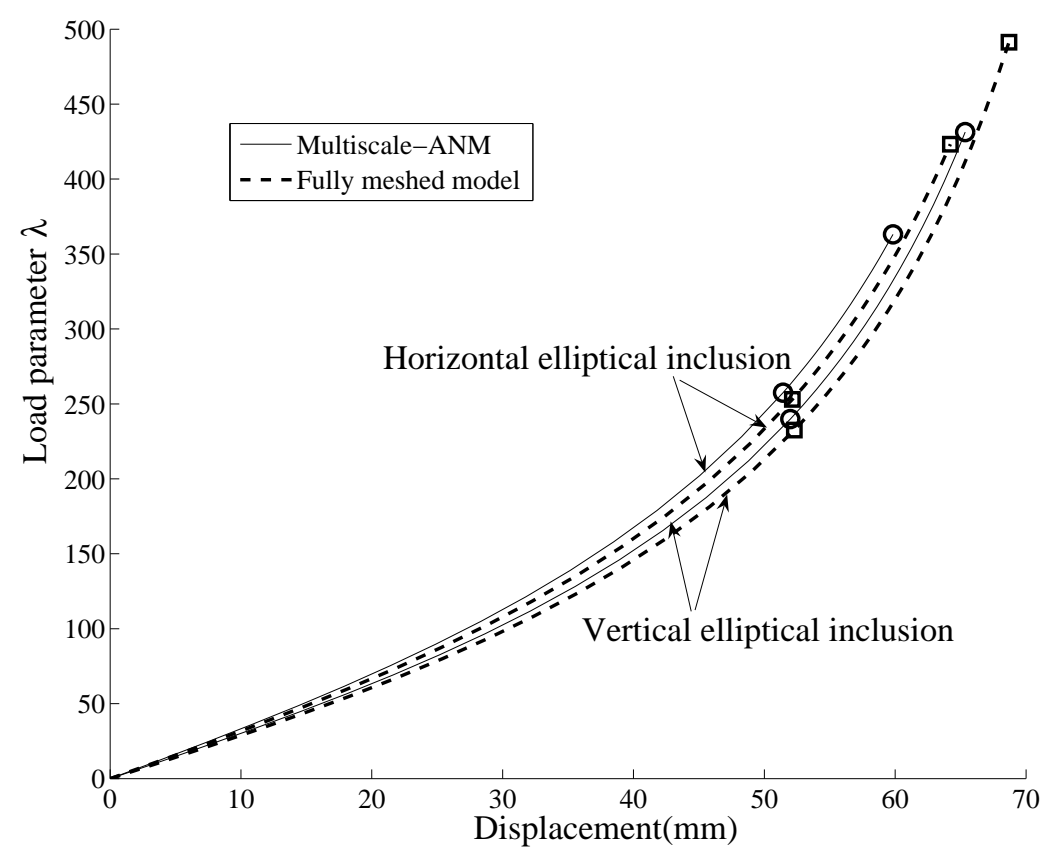

Fig. 9. The load-displacement diagrams of the bending problem for the biphasic RVE with vertical and horizontal elliptical inclusion and the rigidity ratio of 10 , displacement of point $C$.

achieve the response curve.

Figures 4, 6 and 9 allow one to compare the results of global variables, e.g. displacement versus loading parameter, and give satisfactory results. For a complete analysis, we compared local variables, e.g. stresses versus loading parameter at two points $A$ and $B$ exhibiting large deformation for $A$ and large rotations for $B$ (see figures 7 and 8 ). Once more, we can note the high accuracy of the proposed model to compute these different fields.

In the previous calculations, the step ends have been defined by the equation 13 where the macroscopic variables are utilized. Of course, a validity range of the series can be defined in the same way on each microstructure. We have compared the averaged value of these microscopic validity ranges with the macroscopic one and they are very close; e.g. for the biphasic RVE with circular inclusion and rigidity ratio of 10, at first step, the average of all microscopic validity ranges is 392 , and the macroscopic validity range is 426 . Furthermore, the choice of a small accuracy parameter, $\delta=10^{-6}$, makes sure that the solution accuracy is acceptable throughout the step. In particular, figures 7 and 8 show that the local variables are obtained with fine accuracy. 


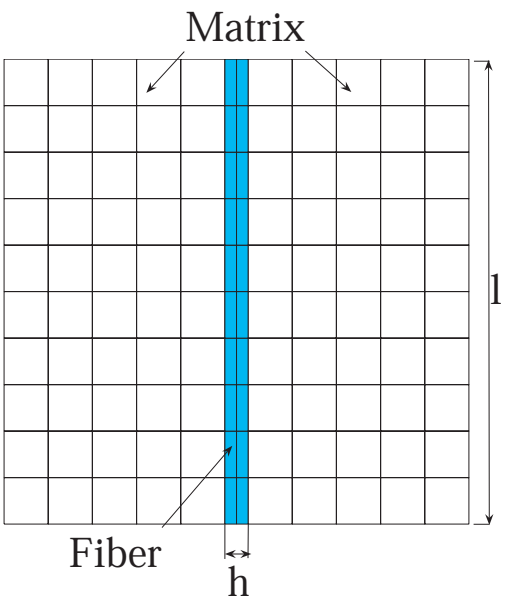

Fig. 10. Elementary cell of fiber reinforced composite material, $\frac{h}{l}=\frac{1}{20}$.

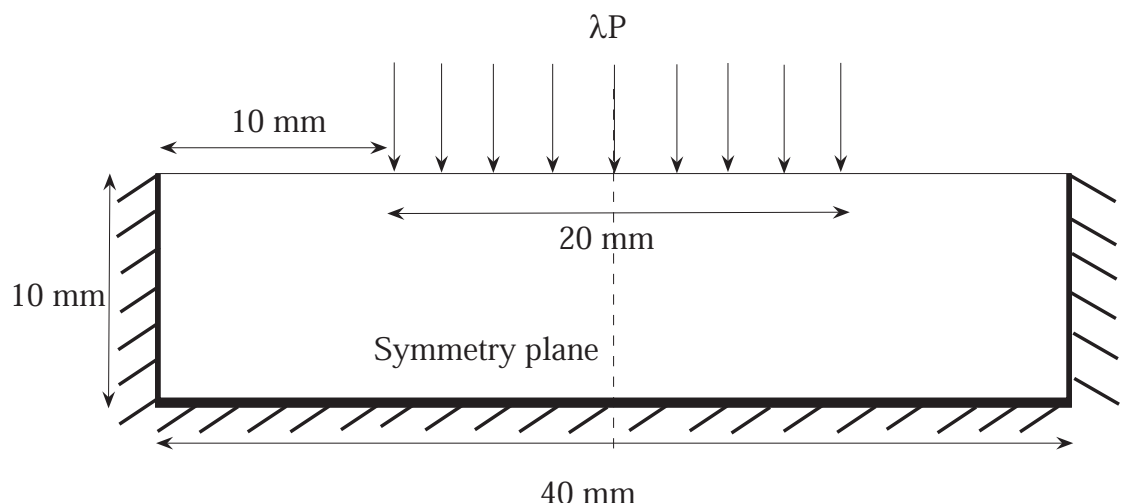

Fig. 11. Geometry and boundary conditions of the rectangular plate made of the fiber composites, $\mathrm{P}=1 \mathrm{~N} / \mathrm{mm}$

\subsection{Micro buckling of a fiber composite}

\subsubsection{Fiber-reinforced composite}

As second example, the compression of a fiber reinforced composite material where instabilities occur at both the macro and micro scales is studied (figure 10). We consider a linear deformation on the boundary of RVE as described in equation (5). The rigidity ratio of fiber per matrix is equal to 1000 $\left(E_{\text {Fiber }} / E_{\text {Matrix }}=1000\right.$ and $\left.E_{\text {Fiber }}=100000 M P a\right)$. The macrostructure is shown in figure 11 which presents a rectangular plate clamped on three edges and submitted to a force distribution $\lambda P$ on the top edge. Because of the symmetry of the problem, only a half of the structure is discretized.

Different RVE can be constructed from the elementary cell shown in figure 10. However, the choice of a suitable RVE is of critical importance. Obviously, one suitable RVE can be found when the average response (or effective prop- 


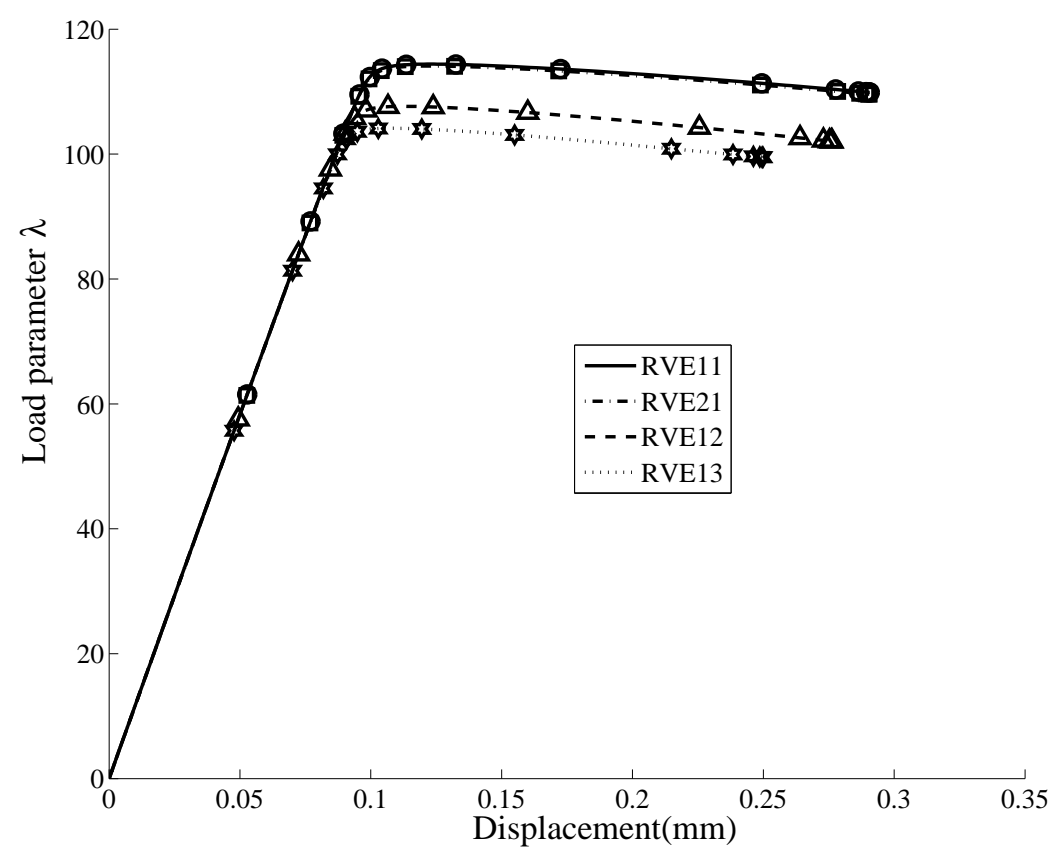

Fig. 12. The load-displacement diagrams of the fiber-reinforced composite problem in compression for different RVE. The different symbols indicate the end of steps.

erties) converges with respect to the RVE's size. In this study, we consider different numbers of elementary cells in the horizontal and vertical directions to construct the RVE and to analyse the overall response of the structure. The load-displacement response for different RVE is presented in Figure 12 which shows the displacement of a point located on the top of macroscopic element 1 (see figure 14). The notation $\mathrm{RVE}_{i j}$ corresponds to a RVE containing $i$ cells in the horizontal direction, and $j$ cells in the vertical direction. Figures 12 and 13 show rather fast convergence of the critical buckling load $\lambda_{C}$ which corresponds to the maximum value of $\lambda$. This convergence is essentially due to the fact that for the chosen example, there is a micro-buckling wavelength. The optimal size of the RVE is related to this wavelength.

Figure 14 shows the deformed shape of half of the macrostructure. Figures 15 and 16 illustrate the buckling of fiber in the microstructure for different types of RVE.

In this example, local instabilities play an important role on the macroscopic response of the structure. This is shown clearly in figure 17: initially (in the portion of the curve preceding point $\mathrm{A}$ ), the macroscopic behaviour is mainly linear. When instabilities occur at the microscopic level, a brutal change (softening) is noticed (portion of the curve following point B). Figure 18 shows the deformed shapes of $\mathrm{RVE}_{13}$ before and after the global buckling load (point $\mathrm{A}$ and point $\mathrm{B}$ in figure 17). There is a large difference between the two deformed microstructural shapes despite the slight variation of the macroscopic load. In this case, this establishes the coincidence between local and global 


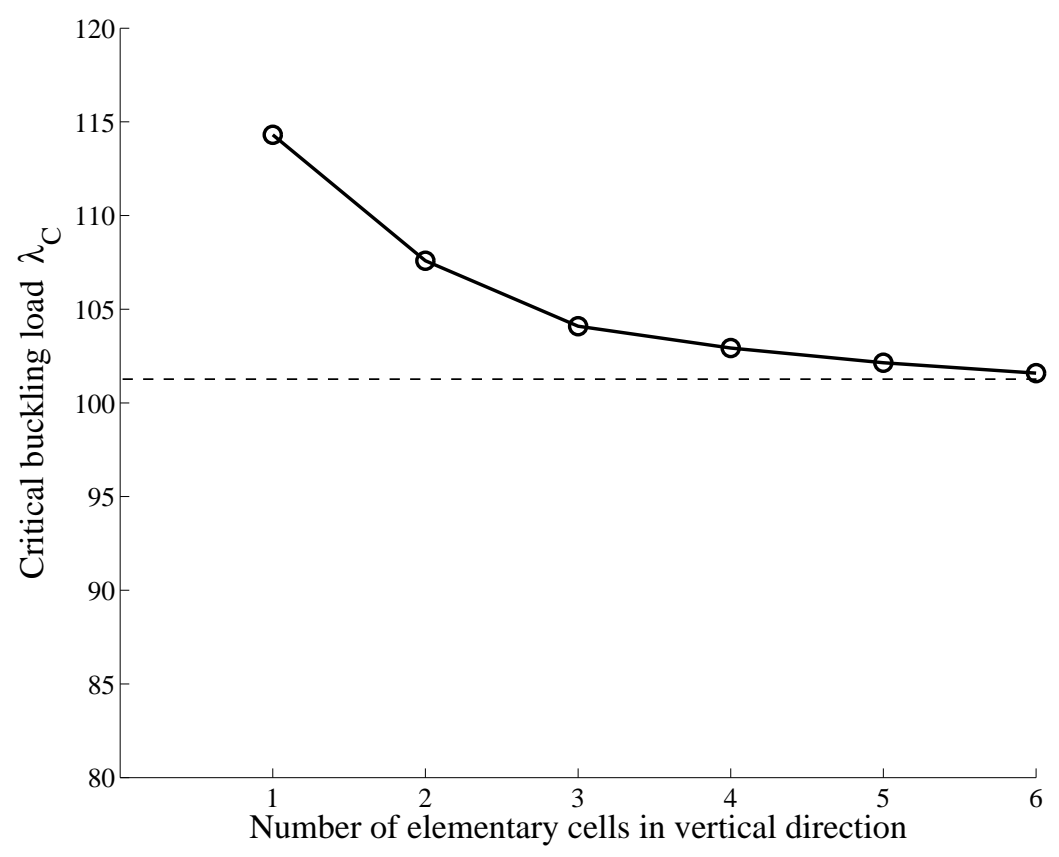

Fig. 13. Convergence of critical load according to the number of elementary cells in vertical direction for the fiber-reinforced composite.

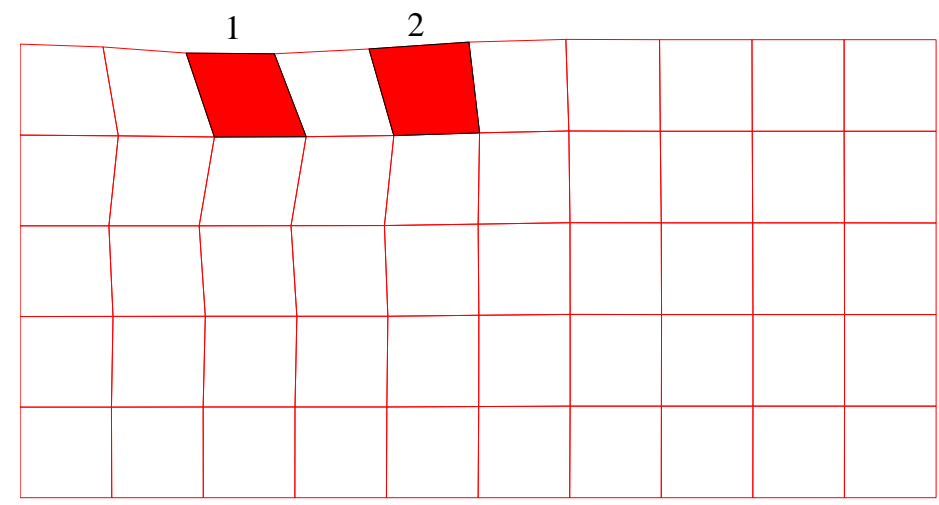

Fig. 14. Deformed shaped of the macrostructure for the fiber-reinforced composite problem.

instabilities.

Fiber microbuckling is a very well known phenomenon that is the basic mechanism to describe the compressive failure of long fiber composites. It is generally described numerically at the microscopic level, as done for instance by Kyriakides et al. [24], Lee and Waas [28], Grandidier et al., [20] and Drapier et al. [12] have considered macroscopic models deduced from computational homogenization in the transverse plane. According to the present computations, the multilevel finite element analysis combined with the asymptotic numerical method (Multiscale-ANM) appears as a new attractive technique to model the 


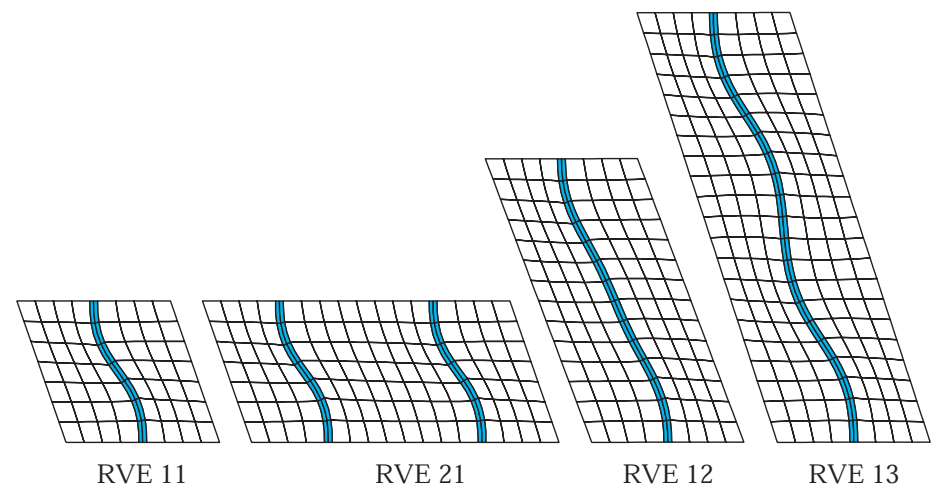

Fig. 15. Deformed shapes of microstructure in the element 1 (see figure 14).

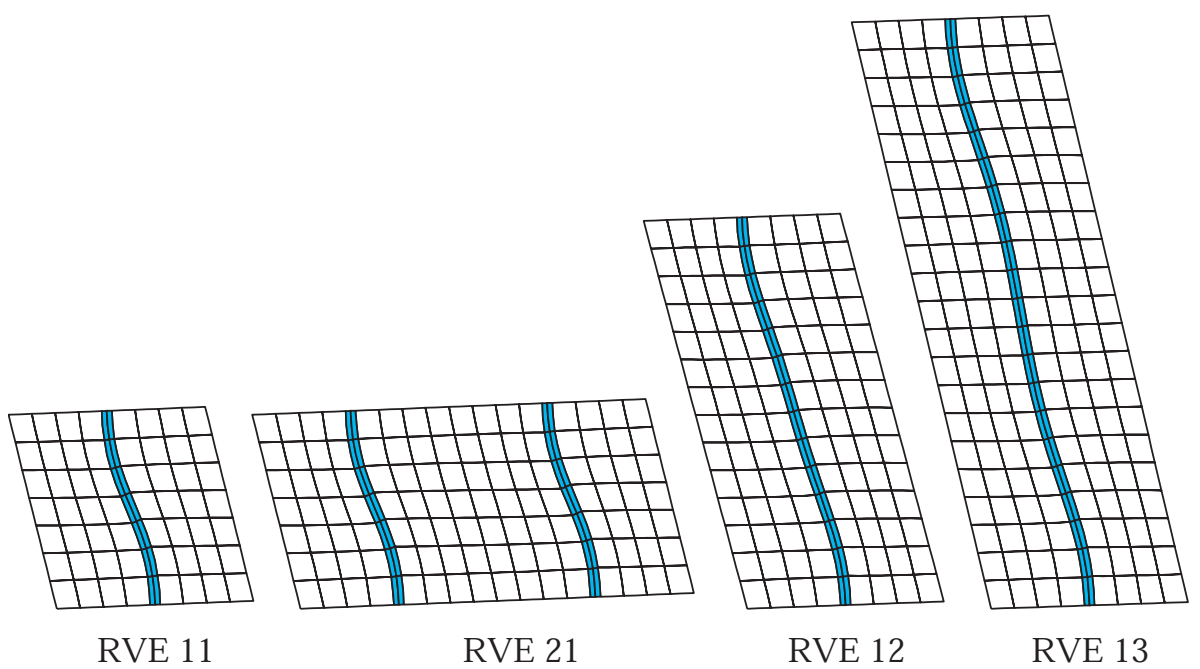

Fig. 16. Deformed shapes of microstructure in the element 2 (see figure 14).

compressive failure of composites. Nevertheless, realistic predictions should account for fiber waviness and plastic behavior of the matrix. 


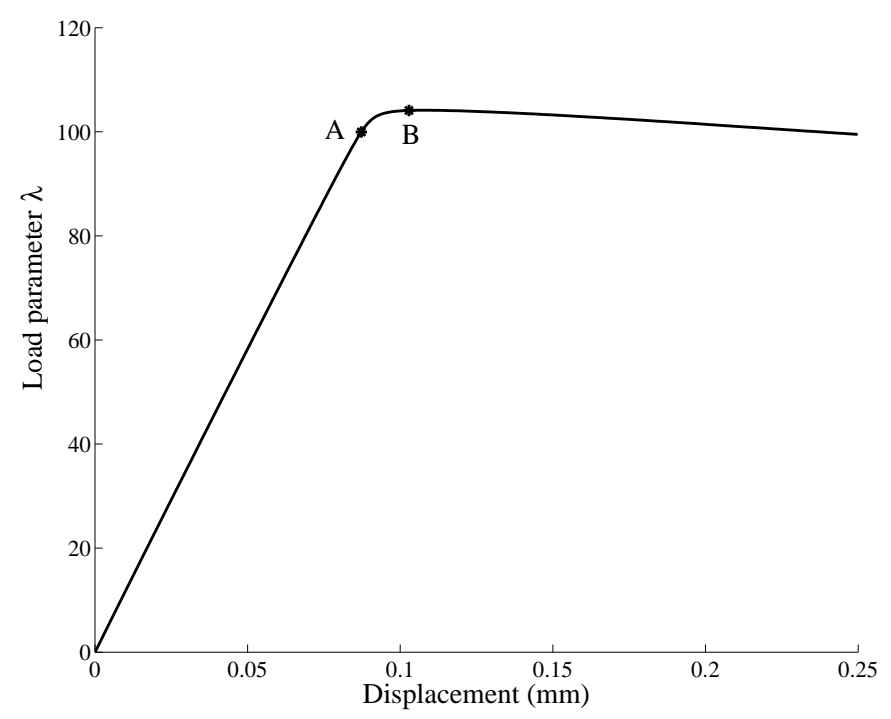

Fig. 17. The load-displacement diagram of the fiber-reinforced problem using $\mathrm{RVE}_{13}$ shown in figure 10 .

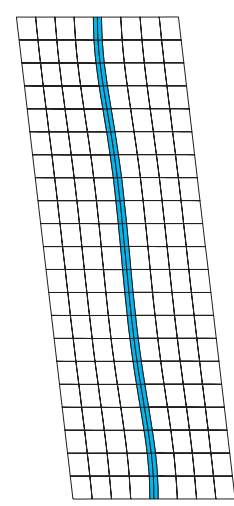

(a)

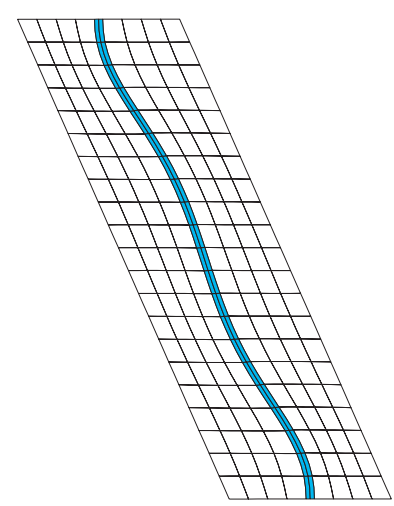

(b)

Fig. 18. Deformed shapes of microstructure in the element 1 (see figure 14) at (a) point $\mathrm{A}$, and (b) point $\mathrm{B}$ (see figure 17), deformation scale $=5$.

\subsubsection{Fiber-weakened composite}

To assess clearly the influence of microscopic instability on the macroscopic one, we consider another microstructure with inclusions weaker than the matrix, which RVE's geometry is depicted in figure 19 a, using the periodic boundary conditions (6). The same macrostructure, macroscopic boundary conditions (figure 11) and mechanical properties as in the previous example are used.

According to the previous calculation, the compression is the main mode applied to the microstructure. Before considering the micro-macro coupling, let us find the suitable RVE by studying a microstructure with different num- 


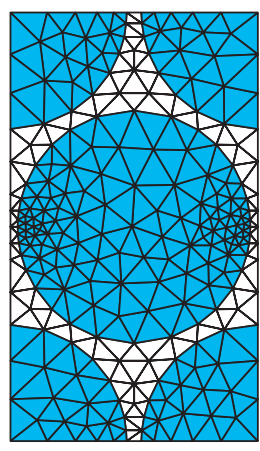

(a)

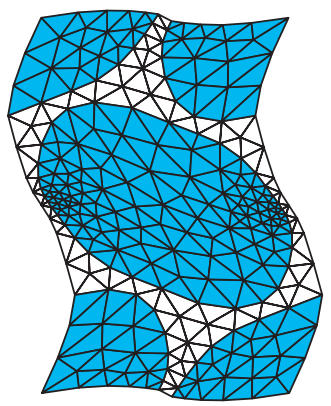

(b)

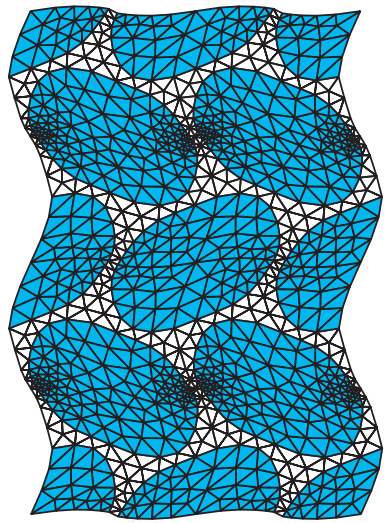

(c)

Fig. 19. (a) Elementary cell of the fiber-weakened composite problem (RVE 11 ); (b) deformed shape of $\mathrm{RVE}_{11}$; and (c) deformed shape of microstructure with four elementary cells $\left(\mathrm{RVE}_{22}\right)$.

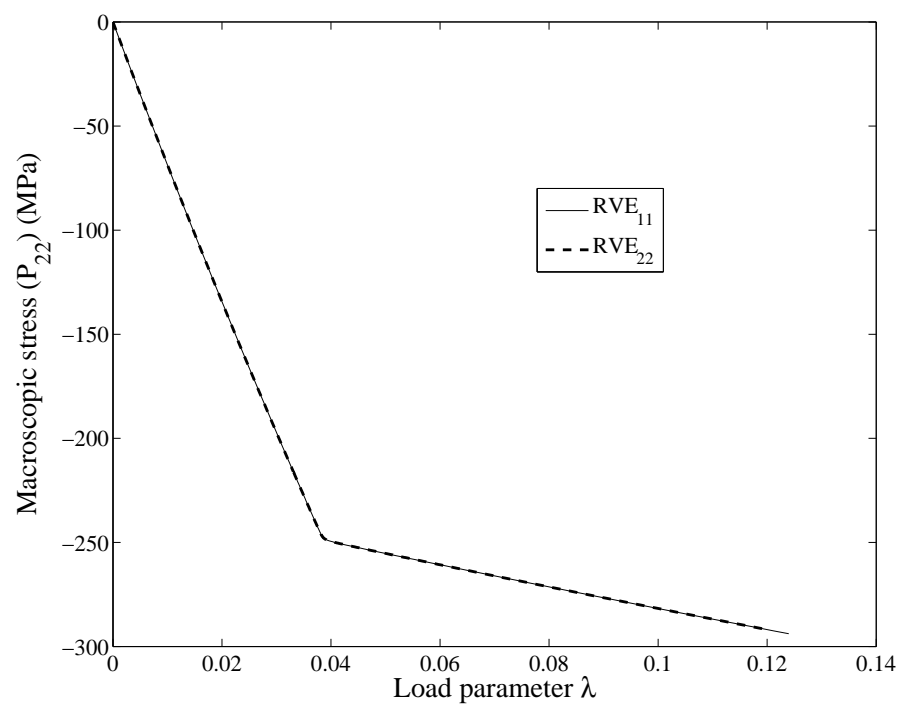

Fig. 20. The stress-load diagrams for the elemntary cells $\mathrm{RVE}_{11}$ and $\mathrm{RVE}_{22}$ described in figure 19.

bers of elementary cells subjected to a macroscopic compression mode with a small shear to initiate the buckling; $\overline{\mathbf{F}}-\mathbf{I}=\left[\bar{F}_{11}-1 ; \bar{F}_{12} ; \bar{F}_{21} ; \bar{F}_{22}-1\right]=$ $[0 ; 0.01 ; 0 ;-1]$ (see equation (6)). Figure 20 presents the averaged stresses $\mathbf{P}_{22}$ versus the loading parameter for $\mathrm{RVE}_{11}$ and $\mathrm{RVE}_{22}$. It shows the buckling of these microstructures and the fast convergence of the responses. Moreover, the deformed shapes of $\mathrm{RVE}_{11}$ and $\mathrm{RVE}_{22}$ are shown in figures $19 \mathrm{~b}$ and $19 \mathrm{c}$, respectively.

We then use our multiscale algorithm to solve the problem of figure 11 with $\mathrm{RVE}_{11}$ as microstructure. Figure 21 shows the displacement of a point located on the top of macroscopic element 1 (figure 14) versus loading parameter. 


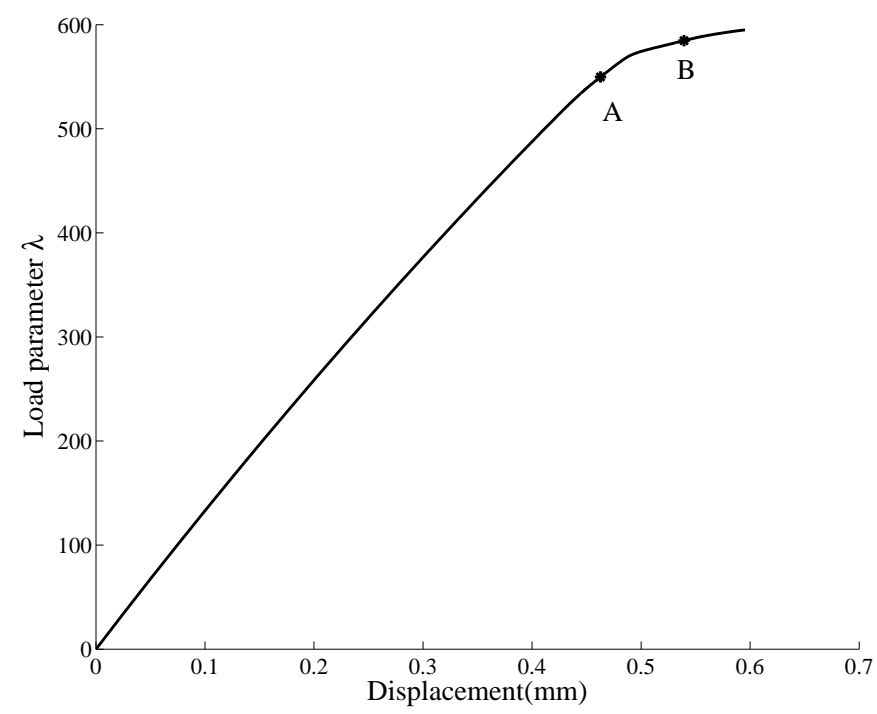

Fig. 21. The load-displacement diagram of the fiber-weakened composite problem for the elemntary cell RVE 11 .

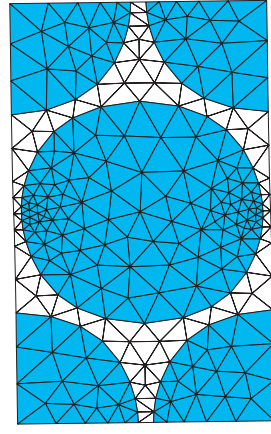

(a)

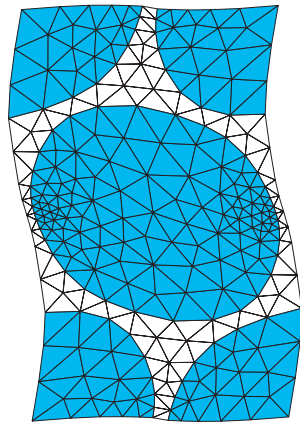

(b)

Fig. 22. Deformed shapes of microstructure in the element 1 (figure 14) at (a) point $\mathrm{A}$, and (b) point B (see figure 21), deformation scale $=1$.

Figure 22 shows the deformed shapes of this microstructure in the element 1 at point $\mathrm{A}$ and point $\mathrm{B}$ (figure 21). As in the previous example, before point $A$, the response is mainly linear. After point B, instabilities at the local level induce a change in the rigidity of the structure.

\section{Conclusion}

We proposed an efficient numerical technique, in the context of heterogeneous materials, combining multilevel finite element analysis $\left(\mathrm{FE}^{2}\right)$ and asymptotic numerical method (ANM) to deal with instabilities which may occur on both the macro and micro levels. This work is considered as the first ANM con- 
tribution which deals with instabilities of heterogeneous materials. We have limited ourselves to linear constitutive relations for the microscale but large displacements are taken into account in both macro and micro levels. Nonlinear constitutive relations can readily be implemented in this model for more realistic applications [62], [63]. Using ANM, the nonlinear problem to be solved is transformed into a sequence of linear problems. Consequently, in the homogenization context, localisation and homogenization tensors can be constructed for each linear local problem. We then compute the tangent stiffness matrix which is the same for all the linear local problems allowing a significant reduction of computation times since only one decomposition of this matrix is needed for the asymptotic steps. The length of each step is a posteriori estimated using the previously computed terms of the series. It allows one to obtain a naturally adaptive step length algorithm. This is not the case for the classical iterative algorithms based on Newton-Raphson procedure. Examples presented in section 4 show that ANM allows to obtain large steps and to handle response curves with complex behaviour. Note that in the ANM framework, it is possible to perform iterative procedures using a high order algorithm for the prediction and a high order algorithm for the correction (see [27]). Furthermore, efficient algorithms have been established to detect bifurcation points and to compute post-buckling response of thin structures [3], [58]. This procedure can be easily implemented in the algorithm proposed in this work. The accuracy and the efficiency of the proposed procedure have been shown through several examples. First, we have validated the proposed algorithm by studying the bending of a rectangular heterogeneous beam. A comparison with the fully meshed model shows that our approach describes accurately the overall behavior of the heterogeneous structure by analyzing global variables as nodal displacement field and local variables as spatial averaging stresses. We then have investigated global and local instabilities by considering the compression of a fiber reinforced composite material. These tests illustrate the capability of our algorithm to deal with instabilities in the context of heterogeneous materials.

Determination of the relevant size of RVE is a major difficulty in the homogenization of heterogeneous materials. The Multiscale-ANM approach, like all of homogenization techniques, can be employed whenever the RVE is well chosen; i.e. the RVE is that for which the overall macro responses converge. However, as indicted in the last example, searching the desirable RVE needs large CPU time and is not always easy. The future directions of this work will take into account nonlinear constitutive relations and surface energy that appear for example in nano materials or imperfectly bonded fibers. 


\section{Appendix}

\subsection{Appendix I}

Expansion of (16) gives:

$$
\int_{\omega}\left(\mathbf{S}_{0}+\sum_{p=1}^{N} a^{p} \mathbf{S}_{p}\right):\left(\boldsymbol{\delta} \mathbf{E}_{0}+\sum_{p=1}^{N} a^{p} \boldsymbol{\delta} \mathbf{E}_{p}\right) d \omega=0
$$

The tensors $\delta \mathrm{E}_{0}$ and $\delta \mathbf{E}_{p}$ are given by expressing $\delta \mathbf{E}$ :

$$
\boldsymbol{\delta} \mathbf{E}=\frac{1}{2}\left(\nabla_{X} \boldsymbol{\delta} \mathbf{u}+\nabla_{X} \boldsymbol{\delta} \mathbf{u}^{T}+\nabla_{X} \mathbf{u}^{T} \nabla_{X} \boldsymbol{\delta} \mathbf{u}+\nabla_{X} \boldsymbol{\delta} \mathbf{u}^{T} \nabla_{X} \mathbf{u}\right)
$$

Introducing the expansion (11) in (28), we obtain $\boldsymbol{\delta} \mathbf{E}=\boldsymbol{\delta} \mathbf{E}_{0}+\sum_{p=1}^{N} a^{p} \boldsymbol{\delta} \mathbf{E}_{p}$ with:

$$
\begin{aligned}
& \boldsymbol{\delta} \mathbf{E}_{0}=\frac{1}{2}\left(\boldsymbol{\nabla}_{X} \boldsymbol{\delta} \mathbf{u}+\nabla_{X} \boldsymbol{\delta} \mathbf{u}^{T}+\nabla_{X} \mathbf{u}_{0}^{T} \boldsymbol{\nabla}_{X} \boldsymbol{\delta} \mathbf{u}+\nabla_{X} \boldsymbol{\delta} \mathbf{u}^{T} \nabla_{X} \mathbf{u}_{0}\right) \\
& =\mathbb{B}: \nabla_{X} \boldsymbol{\delta} \mathbf{u}
\end{aligned}
$$

where $\mathbb{B}$ is a fourth order tensor depending on the initial solution $\mathbf{u}_{0}$ defined by:

$$
\mathbb{B}=\mathbf{I} \underline{\bar{\otimes}} \mathbf{I}+\frac{1}{2}\left(\nabla_{X}^{T} \mathbf{u}_{0} \bar{\otimes} \mathbf{I}+\mathbf{I} \underline{\otimes} \nabla_{X}^{T} \mathbf{u}_{0}\right)
$$

with $(\mathbf{A} \bar{\otimes} \mathbf{B})_{i j k l}=A_{i k} B_{j l},(\mathbf{A} \underline{\otimes} \mathbf{B})_{i j k l}=A_{i l} B_{j k}$ and $(\mathbf{A} \underline{\otimes} \mathbf{B})_{i j k l}=\frac{1}{2}\left(A_{i k} B_{j l}+A_{i l} B_{j k}\right)$ and

$$
\boldsymbol{\delta} \mathbf{E}_{p}=\frac{1}{2}\left(\boldsymbol{\nabla}_{X} \mathbf{u}_{p}^{T} \boldsymbol{\nabla}_{X} \boldsymbol{\delta} \mathbf{u}+\nabla_{X} \boldsymbol{\delta} \mathbf{u}^{T} \boldsymbol{\nabla}_{X} \mathbf{u}_{p}\right)
$$

Now let us express $\mathbf{S}_{p}$. By expanding $\mathbf{S}=\mathbb{C}^{(r)}: \mathbf{E}=\mathbb{C}^{(r)}:\left(\mathbf{E}_{0}+\sum_{p=1}^{N} \mathbf{E}_{p}\right)$, we obtain at order $p$ :

$$
\mathbf{S}_{p}=\mathbb{C}^{(r)}: \mathbf{E}_{p}
$$

To identifify $\mathbf{E}_{p}$ we expand the Green-Lagrange strain (17) that gives at order $p$ :

$$
\mathbf{E}_{p}=\frac{1}{2}\left\{\boldsymbol{\nabla}_{X} \mathbf{u}_{p}+\boldsymbol{\nabla}_{X} \mathbf{u}_{p}^{T}+\boldsymbol{\nabla}_{X} \mathbf{u}_{0}^{T} \boldsymbol{\nabla}_{X} \mathbf{u}_{p}+\boldsymbol{\nabla}_{X} \mathbf{u}_{p}^{T} \boldsymbol{\nabla}_{X} \mathbf{u}_{0}\right\}+\mathbf{E}_{p}^{n l},
$$


where

$$
\begin{aligned}
& \mathbf{E}_{1}^{n l}=0 \\
& \mathbf{E}_{p}^{n l}=\frac{1}{2} \sum_{i=1}^{p-1} \boldsymbol{\nabla}_{X} \mathbf{u}_{i}^{T} \boldsymbol{\nabla}_{X} \mathbf{u}_{p-i}, \quad \forall p=2, \ldots, N .
\end{aligned}
$$

As $\mathbf{E}_{p}$ is linear with respect to $\boldsymbol{\nabla}_{X} \mathbf{u}_{p}$, equation (33) can be rewritten in the form:

$$
\mathbf{E}_{p}=\mathbb{B}: \nabla_{X} \mathbf{u}_{p}+\mathbf{E}_{p}^{n l}=\mathbb{B}: \mathbf{F}_{p}+\mathbf{E}_{p}^{n l}
$$

Then $\mathbf{S}_{p}$ is expressed by

$$
\mathbf{S}_{p}=\mathbb{C}^{(r)}: \mathbf{E}_{p}=\mathbb{C}^{(r)}: \mathbb{B}: \mathbf{F}_{p}+\mathbf{S}_{p}^{n l}, \quad \mathbf{S}_{p}^{n l}=\mathbb{C}^{(r)}: \mathbf{E}_{p}^{n l}
$$

By substituting equation (36) in (27), we obtain equation (18). The explicit forms of the operators involved in the resolution of the sequence of linear problems in equation (18) are given by:

$$
\mathcal{L}\left(\mathbf{u}_{p}, \boldsymbol{\delta} \mathbf{u}\right)=\int_{\Omega}\left\{\mathbf{S}_{0}: \delta \mathbf{E}_{p}+\mathbf{F}_{p}: \mathbb{B}: \mathbb{C}^{(r)}: \delta \mathbf{E}_{0}\right\} d \Omega
$$

and

$$
\mathcal{F}_{p}^{n l}(\boldsymbol{\delta} \mathbf{u})=-\int_{\Omega} \sum_{i=1}^{p-1}\left\{\mathbf{S}_{i}: \delta \mathbf{E}_{p-i}+\mathbf{E}_{p}^{n l}\left(\mathbf{u}_{i}, \mathbf{u}_{p-i}\right): \mathbb{C}^{(r)}: \delta \mathbf{E}_{0}\right\} d \Omega
$$

\subsection{Appendix II}

As each problem at order $p$ is linear, its solution $\mathbf{u}_{p}(\mathbf{X})$ can be decomposed into modes associated with different boundary conditions responses. Here we give an illustration in the $2 \mathrm{D}$ case, though the methodology can be readily extended to three dimensions. We can write:

$$
\begin{aligned}
& \mathbf{u}_{p}(\mathbf{X})=\mathbf{u}_{p}^{l}(\mathbf{X})+\mathbf{u}_{p}^{n l}(\mathbf{X}) \\
& =\bar{F}_{p}^{11} \tilde{\mathbf{u}}^{(11)}(\mathbf{X})+\bar{F}_{p}^{12} \tilde{\mathbf{u}}^{(12)}(\mathbf{X})+\bar{F}_{p}^{21} \tilde{\mathbf{u}}^{(21)}(\mathbf{X})+\bar{F}_{p}^{22} \tilde{\mathbf{u}}^{(22)}(\mathbf{X}) \\
& +\mathbf{u}_{p}^{n l}(\mathbf{X}) \text { in } \omega
\end{aligned}
$$

where $\tilde{\mathbf{u}}^{(i j)}(\mathbf{X})$ are the solutions of the following problems: 
- Linear deformation condition

$$
\left\{\begin{array}{l}
\mathcal{L}\left(\tilde{\mathbf{u}}^{(i j)}, \boldsymbol{\delta} \mathbf{u}\right)=0 \text { in } \omega \\
\tilde{\mathbf{u}}^{(i j)}=\mathbf{X}^{(i j)} \text { on } \partial \omega
\end{array}\right.
$$

with

$$
\begin{aligned}
& \mathbf{X}^{(11)}=\left[\begin{array}{ll}
1 & 0 \\
0 & 0
\end{array}\right] \mathbf{X}, \mathbf{X}^{(12)}=\left[\begin{array}{ll}
0 & 1 \\
0 & 0
\end{array}\right] \mathbf{X}, \\
& \mathbf{X}^{(21)}=\left[\begin{array}{ll}
0 & 0 \\
1 & 0
\end{array}\right] \mathbf{X}, \mathbf{X}^{(22)}=\left[\begin{array}{ll}
0 & 0 \\
0 & 1
\end{array}\right] \mathbf{X}
\end{aligned}
$$

- Periodic condition

$$
\left\{\begin{array}{l}
\mathcal{L}\left(\tilde{\mathbf{u}}^{(i j)}, \boldsymbol{\delta} \mathbf{u}\right)=0 \text { in } \omega \\
\tilde{\mathbf{u}}^{(i j)+}-\tilde{\mathbf{u}}^{(i j)-}=\mathbf{X}^{(i j)+}-\mathbf{X}^{(i j)-} \text { on } \partial \omega
\end{array}\right.
$$

with

$$
\begin{aligned}
& \mathbf{X}^{(11)+}-\mathbf{X}^{(11)-}=\left[\begin{array}{ll}
1 & 0 \\
0 & 0
\end{array}\right]\left(\mathbf{X}^{+}-\mathbf{X}^{-}\right) \\
& \mathbf{X}^{(12)+}-\mathbf{X}^{(12)-}=\left[\begin{array}{ll}
0 & 1 \\
0 & 0
\end{array}\right]\left(\mathbf{X}^{+}-\mathbf{X}^{-}\right) \\
& \mathbf{X}^{(21)+}-\mathbf{X}^{(21)-}=\left[\begin{array}{ll}
0 & 0 \\
1 & 0
\end{array}\right]\left(\mathbf{X}^{+}-\mathbf{X}^{-}\right) \\
& \mathbf{X}^{(22)+}-\mathbf{X}^{(22)-}=\left[\begin{array}{ll}
0 & 0 \\
0 & 1
\end{array}\right]\left(\mathbf{X}^{+}-\mathbf{X}^{-}\right)
\end{aligned}
$$

and $\mathbf{u}_{p}^{n l}(\mathbf{X})$ is the solution of the problem:

$$
\mathcal{L}\left(\tilde{\mathbf{u}}^{(i j)}, \boldsymbol{\delta} \mathbf{u}\right)=\mathcal{F}_{p}^{n l}(\boldsymbol{\delta} \mathbf{u}) \text { in } \omega
$$

The solution $\mathbf{u}_{p}(\mathbf{X})$ can thus be expressed by:

$$
\mathbf{u}_{p}(\mathbf{X})=\mathbb{A}(\mathbf{X}): \overline{\mathbf{F}}_{p}+\mathbf{u}_{p}^{n l}(\mathbf{X})
$$


where $\mathbb{A}(\mathbf{X})$ is a third-order tensor defined by $A_{i j k}=\tilde{u}_{i}^{(j k)}$.

\subsection{Appendix III}

We expand $\mathbf{P}=\mathbf{F S}$ which leads to:

$$
\left(\mathbf{P}_{0}+\sum_{p=1}^{N} a^{p} \mathbf{P}_{p}\right)=\left(\mathbf{F}_{0}+\sum_{p=1}^{N} a^{p} \mathbf{F}_{p}\right)\left(\mathbf{S}_{0}+\sum_{p=1}^{N} a^{p} \mathbf{S}_{p}\right) .
$$

We obtain:

$$
\begin{aligned}
& \mathbf{P}_{1}=\mathbf{F}_{0} \mathbf{S}_{1}+\mathbf{F}_{1} \mathbf{S}_{0}, \\
& \mathbf{P}_{p}=\mathbf{F}_{0} \mathbf{S}_{p}+\mathbf{F}_{p} \mathbf{S}_{0}+\sum_{i=1}^{p-1} \mathbf{F}_{i} \mathbf{S}_{p-i}, \quad \forall p>1 .
\end{aligned}
$$

By using equation (36) and setting $\sum_{i=1}^{p-1} \mathbf{F}_{i} \mathbf{S}_{p-i}=\mathbf{P}_{p}^{* n l}$, we can express $\mathbf{P}_{p}$ as:

$$
\mathbf{P}_{p}=\mathbf{F}_{0}\left\{\mathbb{C}^{(r)}: \mathbb{B}: \mathbf{F}_{p}+\mathbf{S}_{p}^{n l}\right\}+\mathbf{F}_{p} \mathbf{S}_{0}+\mathbf{P}_{p}^{* n l},
$$

which can be rewritten in the form:

$$
\mathbf{P}_{p}=\mathbb{H}^{(r)}: \mathbf{F}_{p}+\mathbf{F}_{0} \mathbf{S}_{p}^{n l}+\mathbf{P}_{p}^{* n l}
$$

Using (22), we obtain:

$$
\mathbf{P}_{p}=\mathbb{H}^{(r)}: \mathbb{A}_{, \mathbf{X}}: \overline{\mathbf{F}}_{p}+\mathbb{H}^{(r)}: \mathbf{u}_{p, \mathbf{X}}^{n l}+\mathbf{F}_{0} \mathbf{S}_{p}^{n l}+\mathbf{P}_{p}^{* n l}
$$

By setting $\mathbf{P}_{p}^{n l}=\mathbb{H}^{(r)}: \mathbf{u}_{p, \mathbf{X}}^{n l}+\mathbf{F}_{0} \mathbf{S}_{p}^{n l}+\mathbf{P}_{p}^{* n l}$ and $\mathbb{L}=\mathbb{H}^{(r)}(\mathbf{X}): \mathbb{A}_{, \mathbf{X}}(\mathbf{X})$, we finally obtain:

$$
\mathbf{P}_{p}=\mathbb{L}: \overline{\mathbf{F}}_{p}+\mathbf{P}_{p}^{n l}
$$

\section{References}

[1] R. Abeyaratne, N. Triantafyllidis, An investigation of localization in a porous elastic material using homogenization theory, Journal of Applied Mechanics 51 (1984) 481-486.

[2] W. Aggoune, H. Zahrouni, M. Potier-Ferry, Asymptotic numerical method for unilateral contact, International Journal for Numerical Method in Engineering 
68 (2006) 605-631.

[3] E.H. Boutyour, H. Zahrouni, M. Potier-Ferry, M. Boudi, Bifurcation points and bifurcated branches by an asymptotic numerical method and padé approximants, International Journal for Numerical Methods in Engineering 60 (2004) 1987-2012.

[4] J.M. Cadou, B. Cochelin, M. Potier-Ferry, A numerical method for the computations of bifurcation points in fluid mechanics. European Journal of Mechanics-B 25 (2006) 234-254.

[5] T. Christman, A. Needleman, S. Suresh, An experimental and numerical study of deformation in metal-ceramic composites, Acta Metallurgica 37 (1989) 30293050 .

[6] B. Cochelin, A path following technique via an asymptotic numerical method. Computers and Structures 53 (1994) 1181-1192

[7] B. Cochelin, N. Damil, M. Potier-Ferry, Méthode asymptotique numrique, Hermès Science Publications, 2007.

[8] M.A. Crisfield, Non-Linear Finite Element Analysis of Solids and Structures: vol. 1, Wiley, Chichester, 1991.

[9] N. Damil, M. Potier-Ferry, A new method to compute perturbed bifurcation: application to the buckling of imperfect elastic structures, International Journal for Engineering Science 26 (1990) 943-957.

[10] G. deBotton, I. Hariton, E.A. Socolsky, Neo-Hookean fiber reinforced composites in finite elasticity, Journal of the Mechanic and Physics of Solids $54(2006) 533-559$.

[11] J. Descamps, H.L. Cao, M. Potier-Ferry, An asymptotic numerical method to solve large strain viscoplastic problems. Computational plasticity, Fundamentals and Applications, C.I.M.N.E. vol. 1 (1997) 393-400, D.R.J. Owen, E. Oñate and E. Hinton (Eds.), Barcelona.

[12] S. Drapier, J.C. Grandidier, M. Potier-Ferry, A structural approach of plastic microbuckling in long fibre composites: comparison with theoretical and experimental results. International Journal of Solids and Structures 38 (2001) 3877-3904.

[13] K. Erami, N. Ohno, D. Okumura, Long-wave in-plane buckling of elastoplastic square honeycombs, International Journal of Plasticity 22 (2006) 1569-1585.

[14] A. Eriksson, Derivatives of tangential stiffness matrices for equilibrium path descriptions, International Journal for Numerical Methods in Engineering 32 (1991) 1093-1113.

[15] F. Feyel, J.L. Chaboche, FE ${ }^{2}$ multiscale approach for modeling the elastoviscoplastic behavior of long fiber $\mathrm{SiC} / \mathrm{Ti}$ composite materials, Computer Methods in Applied Mechanics and Engineering 183 (2000) 309-330. 
[16] F. Feyel, A multilevel finite element method $\left(\mathrm{FE}^{2}\right)$ to describe the response of highly non-linear structures using generalized continua, Computer Methods in Applied Mechanics and Engineering 192 (2003) 3233-3244.

[17] G. Geymonat, S. Muller, N. Triantafyllidis, Homogenization of nonlinearly elastic materials: microscopic bifurcation and macroscopic loss of rank-one convexity, Archive for Rational Mechanics and Analysis 122 (1993) 231-290.

[18] S. Ghosh, K. Lee, P. Raghavan, A multilevel computational model for multiscale damage analysis in composite and porous media, International Journals of Solids and Structures 38 (2001) 2335-2385.

[19] L. Gong, S. Kyriakides, N. Triantafyllidis, On the stability of Kelvin cell foams under compressive loads, Journal of the Mechanics and Physics of Solids 53 (2005) 771-794

[20] J.C. Grandidier, G. Ferron, M. Potier-Ferry, Microbuckling and strength in long-fiber composites - theory and experiments, International Journal of Solids and Structures 29 (1992) 1753-1761

[21] Z. Hashin, The elastic moduli of heterogeneous materials, Journal of Applied Mechanics 29 (1962) 143-150.

[22] R. Kouhia, Techniques for the analysis of non-linear systems with applications to solids and structural mechanics, Thesis. In the series 'Acta Polytechnica Scandinavia', Espoo, Finland, 1999.

[23] V.G. Kouznetsova, M.G.D. Geers, W.A.M. Brekelmans, Multi-scale second order computational homogenization pf multi-phase materials: a nested finite element solution strategy, Computer Methods in Applied Mechanics and Engineering 193 (2004) 5525-5550.

[24] S. Kyriakides, R. Arseculeratne, E.J. Perry, K.M. Liechti, On the compressive failure of fiber-reinforced composites. International Journal of Solids and Structures 32 (1995) 689-738.

[25] P. Ladevèze, Nonlinear computational structural mechanics: new approaches and non-incremental methods of calculation, Springer-Verlag, Berlin, 1999.

[26] P. Ladevèze, O. Loiseau, D. Dureisseix, A micro-macro and parallel computational strategy for highly heterogeneous structures, International Journal for Numerical Methods in Engineering 52 (2001) 121-138.

[27] H. Lahmam, J.M. Cadou, H. Zahrouni, N. Damil, M. Potier-Ferry, High-order predictor-corrector algorithms, International Journal for Numerical Methods in Engineering 55 (2002) 685-704.

[28] S.H. Lee, A.M. Waas, Compressive response and failure of fiber reinforced unidirectional composites, International Journal of Fracture 100 (1999) 275-306.

[29] O. Lopez-Pamies, P. Ponte Castañeda, Second-order estimated for the macroscopic response and loss of ellipticity in porous rubbers at large deformations, Journal of Elasticity 76 (2005) 247-287. 
[30] O. Lopez-Pamies, P. Ponte Castañeda, On the overall behavior, microstructure evolution, and macroscopic stability in reinforced rubbers at large deformations: I- Theory, Journal of the Mechanics and Physics of Solids 54 (2006) 807-830.

[31] O. Lopez-Pamies, P. Ponte Castañeda, On the overall behavior, microstructure evolution, and macroscopic stability in reinforced rubbers at large deformations: II- Application to cylindrical fibers, Journal of the Mechanics and Physics of Solids 54 (2006) 831-863.

[32] O. Lopez-Pamies, P. Ponte Castañeda, Homogenization-based constitutive models for porous elastomers and implications for macroscopic instabilities: IAnalysis, Journal of the Mechanics and Physics of Solids 55 (2007) 1677-1701.

[33] O. Lopez-Pamies, P. Ponte Castañeda, Homogenization-based constitutive models for porous elastomers and implications for macroscopic instabilities: IIResults, Journal of the Mechanics and Physics of Solids 55 (2007) 1702-1728.

[34] J.C. Michel, O. Lopez-Pamies, P. Ponte Castanñeda, N. Triantafyllidis, Microscopic and macroscopic instabilities in finitely strained porous elastomers, Journal of the Mechanics and Physics of Solids 55 (2007) 900-938.

[35] J.C. Michel, H. Moulinec, P. Suquet, Effective properties of composite materials with periodic microstructure: a computational approach, Computer Methods in Applied Mechanics and Engineering 172 (1999) 109-143.

[36] C. Miehe, J. Schroder, M. Becker, Computational homogenization analysis in finite elasticity: material and structural instabilities on the micro- and macroscales of periodic composites and their interactions, Computer Methods in Applied Mechanics and Engineering 191 (2002) 4971-5005.

[37] C. Miehe, Computational micro-to-macro transitions for discretized microstructures of heterogeneous materials at finite strains based on the minimization of averaged incremental energy, Computer Methods in Applied Mechanics and Engineering 192 (2003) 559-591.

[38] E. Monteiro, J. Yvonnet J., Q.-C. He, Computational Homogenization for nonlinear conduction in heterogeneous materials using model reduction, Computational Materials Science 42 (2008) 704-712.

[39] S. Muller, Homogenization of non-convex integral functional and cellular elastic materials, Archive for Rational Mechanics and Analysis 99 (1987) 189-212.

[40] S. Nemat-Nasser, M. Hori, Micromechanics: Overall properties of heterogeneous materials, North-Holland Series, Applied Mathematics and Mechanics 37, NorthHolland, Amsterdam, 1993.

[41] M. Nestorović, N. Triantafyllidis, Onset of failure in finitely strained layered composites subjected to combined normal and shear loading, Journal of the Mechanics and Physics of Solids 52 (2004) 941-974.

[42] N. Ohno, D. Okumura, H. Noguchi, Microscopic symmetric bifurcation condition of cellular solids based on a homogenization theory of finite 
deformation, Journal of the Mechanics and Physics of Solids 52 (2002) 11251153.

[43] N. Ohno, D. Okumura, T. Niikawa, Long-wave buckling of elastic square honeycombs subject to in-plane biaxial compression, International Journal of Mechanical Sciences 46 (2004) 1697-1713.

[44] D. Okumura, N. Ohno, H. Noguchi, Post-buckling analysis of elastic honeycombs subject to in-plane biaxial compression, International Journal of Solids and Structures 39 (2002) 3487-3503.

[45] D. Okumura, N. Ohno, H. Noguchi, Elastoplastic microscopic bifurcation and post-bifurcation behavior of periodic cellular solids, Journal of the Mechanics and Physics of Solids 52 (2004) 641-666.

[46] D. Okumura, A. Okada, N. Ohno, Buckling behavior of Kelvin open-cell foams under [ [ $\left.\begin{array}{lll}0 & 0 & 1\end{array}\right],\left[\begin{array}{lll}0 & 1 & 1\end{array}\right]$ and [ $\left.\begin{array}{lll}1 & 1 & 1\end{array}\right]$ compressive loads, International Journal of Solids and Structures 45 (2008) 3807-3820.

[47] P. Ponte Castañeda, P. Suquet, Nonlinear composites, Advances in Applied Mechanics, Academic Press, New York 34 (1998) 171-302.

[48] P. Ponte Castañeda, Second-order homogenization estimates for nonlinear composites incorporating field fluctuations-I. Theory, Journal of the Mechanic and Physics of Solids 50 (2002) 737-757.

[49] E. Riks, Some computational aspects of the stability analysis of nonlinear structures, Computer Methods in Applied Mechanics and Engineering 47 (1984) 219-259.

[50] M.W. Schraad, N. Triantafyllidis, Scale effects in media with periodic and nearly periodic microstructures, II-Failure mechanisms, Journal of Applied Mechanics 64 (1997) 763-771.

[51] R. Smit, W. Brekelmans, H. Meijer, Prediction of the mechanical behavior of nonlinear heterogeneous systems by multi-level finite element modeling, Computer Methods in Applied Mechanics and Engineering 155 (1998) 181-192.

[52] P. Suquet, Elements of homogenization for inelastic solid mechanics, in: E. Sanchez-Palenzia, A. Zaoui (Eds.), Homogenization Techniques for composites Materials, Lecture Notes in Physics, Vol. 272 (Springer, Berlin, 1987) 193-278.

[53] K. Terada, N. Kikuchi, A class of general algorithms for multi-scale analysis of heterogeneous media, Computer Methods in Applied Mechanics and Engineering 190 (2001) 5427-5464.

[54] N. Triantafyllidis, S.G. Bardenhagen, The influence of scale size on the stability of periodic solids and the role of associated higher order gradient continuum models, Journal of the Mechanics and Physics of Solids 44 (1996) 1891-1928.

[55] N. Triantafyllidis, B.N. Maker, On the comparison between microscopic and macroscopic instability mechanisms in a class of fiber-reinforced composites, Journal of Applied Mechanics 52 (1985) 794-800. 
[56] N. Triantafyllidis, M.W. Schraad, Onset of failure in aluminium honeycombs under general in-plane loading, Journal of the Mechanics and Physics of Solids 46 (1998) 1089-1124.

[57] N. Triantafyllidis, M.D. Nestorović, M.W. Schraad, Failure surfaces for finitely strained two-phase periodic solids under general in-plane loading, Journal of Applied Mechanics 73 (2006) 505-515.

[58] P. Vannucci, B. Cochelin, N. Damil, M. Potier-Ferry, An asymptotic-numerical method to compute bifurcated branches, International Journal for Numerical Methods in Engineering 41 (1998) 1365-1389.

[59] J.R. Willis, Variational and related methods for the overall properties of composites, in: Advances in Applied Mechanics, Vol. 21, Academic Press, New York (1981) 1-78.

[60] J. Yvonnet, Q.C. He, The Reduced Model Multiscale Method (R3M) for the non-linear homogenization of hyperelastic media at finite strains, Journal of Computational Physics 223 (2007) 341-368.

[61] J. Yvonnet, H. Zahrouni, M. Potier-Ferry, A model reduction method for the post-buckling analysis of cellular microstructures, Computer Methods in Applied Mechanics and Engineering 197 (2007) 265-280.

[62] H. Zahrouni, W. Aggoune, J. Brunelot, M. Potier-Ferry, Asymptotic numerical method for strong nonlinearities, Revue Européenne des Eléments Finis 118 (2004) 13-97.

[63] H. Zahrouni, B. Braikat, N. Damil, M. Potier-Ferry, Solving plasticity problems by a perturbation technique, Proceedings in Applied Mathematics and Mechanics 5 (2005) 455-456.

[64] H. Zahrouni, B. Cochelin, M. Potier-Ferry, Computing finite rotations of shells by an asymptotic-numerical method, Computer Methods in Applied Mechanics and Engineering 175 (1999) 71-85. 